\title{
Time-resolved Coulomb-explosion imaging of nuclear wave-packet dynamics induced in diatomic molecules by intense few-cycle laser pulses
}

\author{
I. A. Bocharova, ${ }^{1, *}$ A. S. Alnaser, ${ }^{2}$ U. Thumm, ${ }^{1}$ T. Niederhausen,${ }^{3}$ D. Ray, ${ }^{1}$ C. L. Cocke,,${ }^{1}$ and I. V. Litvinyuk ${ }^{1,4, \dagger}$ \\ ${ }^{1}$ J. R. Macdonald Laboratory, Kansas State University, Kansas 66506, USA \\ ${ }^{2}$ Department of Physics, The American University of Sharjah, Sharjah, UAE \\ ${ }^{3}$ Departamento de Química, C-IX, Universidad Autónoma de Madrid, ES-28049 Madrid, Spain \\ ${ }^{4}$ Centre for Quantum Dynamics, Griffith University, Nathan, Queensland 4111, Australia
}

(Received 16 September 2010; published 31 January 2011)

\begin{abstract}
We studied the nuclear dynamics in diatomic molecules $\left(\mathrm{N}_{2}, \mathrm{O}_{2}\right.$, and $\left.\mathrm{CO}\right)$ following their interaction with intense near-IR few-cycle laser pulses. Using Coulomb-explosion imaging in combination with the pump-probe approach, we mapped dissociation pathways of those molecules and their molecular ions. We identified all symmetric and asymmetric breakup channels for molecular ions up to $\mathrm{N}_{2}^{5+}, \mathrm{O}_{2}^{4+}$, and $\mathrm{CO}^{4+}$. For each of those channels we measured the kinetic energy release (KER) spectra as a function of delay between the pump and probe pulses. For both $\mathrm{N}_{2}$ and $\mathrm{O}_{2}$ the asymmetric $(3,1)$ channel is only observed for short $(<20 \mathrm{fs})$ delays and completely disappears after that. We interpret this observation as a signature of electron localization taking place in dissociating molecular tri-cations when their internuclear separation reaches about 2.5 times the equilibrium bond length. This is a direct confirmation that electron localization plays an essential role in the universal mechanism of enhanced ionization in homonuclear diatomic molecules. Using classical and quantum mechanical simulations of the time-dependent KER spectra, we identify the pathways and intermediate states involved in the laser-induced dissociation of those molecules.
\end{abstract}

DOI: 10.1103/PhysRevA.83.013417

PACS number(s): $33.80 . \mathrm{Rv}, 33.80 . \mathrm{Wz}, 33.20 . \mathrm{Xx}, 31.50 .-\mathrm{x}$

\section{INTRODUCTION}

The interaction of intense near-IR few-cycle laser pulses with molecules is a subject of significant current interest. Even the simplest diatomic molecule with its additional nuclear degrees of freedom presents a much higher level of complexity than an atom. The molecules can rotate, vibrate, dissociate, and be aligned or oriented. In addition to that, all the strongfield phenomena discovered and studied in atoms-excitation, single and multiple ionization, high harmonic generationdepend nontrivially on the molecular structure (bond lengths and electronic wave functions) and orientation with respect to the laser polarization. With most molecular processes occurring on time scales from a few to hundreds of femtoseconds, femtosecond laser pulses focused to achieve electric field strengths comparable to those inside molecules offer an ideal tool to interrogate, manipulate, and control molecules.

Among molecules, light and heavy hydrogen and their molecular ions remain the most popular and widely studied targets. These simple prototypical systems exhibit a full range of molecular strong-field phenomena, while remaining the only ones amenable to credible $a b$ initio theoretical treatment. In particular, such phenomena as bond softening [1-4] and charge-resonance enhanced ionization [5] (CREI) were first discovered, studied, and understood in hydrogen. Hydrogen was also the molecule in the seminal "molecular clock" experiments $[6,7]$.

Compared to atoms, diatomic molecules like nitrogen have additional degrees of freedom that make their dynamics in the laser field much more complicated. Alignment, symmetric

\footnotetext{
*Current address: Lawrence Berkeley National Laboratory, Berkeley, CA.

†i.litvinyuk@griffith.edu.au
}

and asymmetric charge breakup, enhanced ionization at critical internuclear distance, and a variety of dissociation pathways associated with different electronic states of the parent molecular ions come into play. Many recent studies were dedicated to the study of field-induced alignment $[8,9]$, the angular dependence of the ionization rate [10], and symmetric and asymmetric molecular fragmentation with a single laser pulse $[11,12]$. These experiments were traditionally conducted by measuring the time of flight and/or kinetic energy release (KER) of the atomic fragments, assuming that this energy originated from Coulomb repulsion of the nuclei. The studies showed that the internuclear distance at the moment of separation was about the same regardless of the charge state or even the molecule. It was approximately equal to 2-3 times $R_{e}$ ( $R_{e}$ is the equilibrium bond length of a neutral molecule in its ground electronic and vibrational state). The experimental KER of the fragments was found to be low compared to the Coulomb kinetic energy for laser pulses of 20 fs and longer for nitrogen as well as for several other diatomic molecules [11-14]. These observations are consistent with the theoretical ionization-rate dependence on the internuclear distance and enhanced ionization at a critical internuclear separation [10]. The traditional approach with a single long pulse does not give an answer to the question of how exactly molecular expansion occurs and contains no information on the ionization and dissociation pathways. Observation of the dynamics of internuclear motion requires femtosecond time resolution. Understanding the mechanisms of enhanced ionization, the symmetric and asymmetric dissociation and excitation of molecular and atomic ions may involve many nuclear and electronic degrees of freedom and are only possible by probing the change in the molecular structure as a function of time using ultrashort pulses [15]. While longer ( $>20 \mathrm{fs}$ ) pulses are sufficient to map slower rotational dynamics in molecules like $\mathrm{N}_{2}$ and $\mathrm{O}_{2}$, the vibrational and dissociative dynamics occur on 
a much faster time scale and therefore require shorter pulses ( $<10 \mathrm{fs}$ ) to map nuclear motion and to avoid the enhanced ionization at a critical distance [10].

In our experiments we measured the KER of charge symmetric and asymmetric Coulomb breakup of homonuclear $\mathrm{N}_{2}$ and $\mathrm{O}_{2}$ and heteronuclear $\mathrm{CO}$ diatomic molecules as a function of time. This was done by ionizing and exciting the molecules with an IR laser pulse from their neutral ground states to unknown excited electronic states of the molecular ions on which the nuclear dynamics would be observed. Then a second pulse was employed to project an evolving nuclear wave packet onto a known Coulomb repulsive state. Both pulses were shorter than $10 \mathrm{fs}$, so the changes in the molecular orientation and internuclear separation during the interaction with the pulse were very small. Using the Coulomb-explosion technique [16] and our pump-probe experiment, we mapped the complete dissociative nuclear dynamics of those molecules following their interaction with the laser pulse. In this paper we also present classical and quantum models which describe a general way to simulate the dynamics of a nuclear wave packet and to identify parent molecular ions and relevant electronic states that determine the molecular dynamics of a diatomic molecule.

\section{EXPERIMENTAL SETUP}

In our experiment we used a coincident momentum imaging technique known as COLTRIMS [17] and the pump-probe approach for studying time-resolved molecular dynamics. The setup is schematically shown in Fig. 1. The laser pulse generated by a Ti:sapphire amplifier with a repetition rate of $1 \mathrm{kHz}$, central wavelength of $800 \mathrm{~nm}$, and duration of $35 \mathrm{fs}$ (full width at half maximum, FWHM) was compressed to about 8 fs with a hollow core Ne-filled fiber and a set of chirped mirrors (Femtolasers). Two germanium plates set at the Brewster angle together with a half-wave plate located before the fiber were used to control intensity of the laser field. Pump and probe pulses were produced with a Mach-Zehnder interferometer; the delay was controlled by a computer-controlled translation stage in the probe arm of the interferometer. To vary the intensity and diameter of the pump pulse we had a variable aperture in the pump arm of the interferometer. The peak intensity of the pulses was calibrated

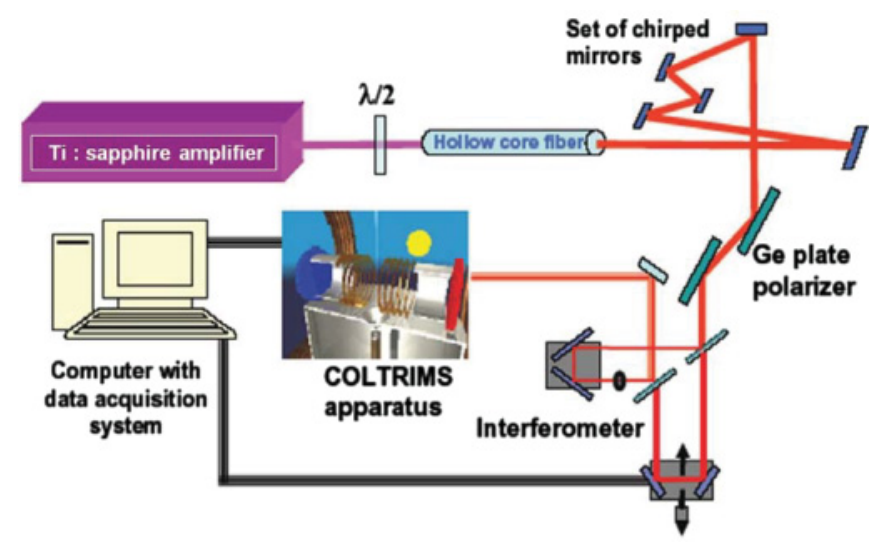

FIG. 1. (Color online) Schematic of the experimental setup. in situ by the method described by Alnaser et al. [18]. The peak intensity of the pulses varied from $8 \times 10^{14}$ to $5 \times$ $10^{15} \mathrm{~W} \mathrm{~cm}{ }^{-2}$. The polarization of the laser field after the germanium plates was horizontal (parallel to the time-of-flight axis of the spectrometer).

Following the interferometer, the pump and probe pulses were overlapped in space and time at zero delay and directed into the UHV COLTRIMS chamber. The chamber design is described in detail elsewhere [13]. Inside the chamber the laser beams were focused by a spherical mirror onto the supersonic target gas jet which was also orthogonal to the time-of-flight axis. The pump pulse ionizes and excites the neutral molecules inside the interaction volume. The probe pulse comes after a certain variable delay to interrogate the dynamics by the Coulomb explosion of the molecular ion. Molecular fragments are carried to the time- and position-sensitive detector (Roentdek) by a uniform electric field of 10-30 V $\mathrm{cm}^{-1}$ applied across the spectrometer. The timing and position information, along with the known value of the electric field and travel distance inside the spectrometer, was used to measure the KER of each pair of ions. To analyze the correlated (coming from the same molecule) ion pairs we only selected pairs of fragments with total momentum close to zero.

\section{SIMULATIONS}

\section{A. Classical model}

Our experimental results can be simulated most easily using purely classical equations of motion and available potential energy curves from the literature. We assume that one (or more) electron is removed by a pump pulse from a neutral molecule in its ground state at the equilibrium internuclear distance. Then, in the classical picture, the "particle" with mass equal to the reduced mass of the nuclei starts moving with zero initial velocity in the potential of the molecular ion. For $\mathrm{N}_{2}$ molecular ions, this potential $[19,20]$ can be purely repulsive, may possess a single minimum, or can be more complicated in shape (Fig. 2). We simulate the subsequent motion of the particle separately in each of these potentials. This motion is followed by a consecutive Coulomb explosion induced by the delayed probe pulse. Ultimately, we obtain a kinetic energy release for the explosion fragments as a function of time delay that can be compared with our experimental spectra. In the experiment the probe pulse comes with various delays and breaks the molecular ion into two ionic atomic fragments. Thus, to simulate the time dependence of the final kinetic energy, we have to add the Coulomb repulsion energy of the resulting fragments to the kinetic energy the particle gained on the ionic potential before the arrival of the probe pulse.

To find out which of the many dissociation pathways is operational in our experimental conditions, we have to make an educated guess and try out a number of different potential curves as intermediate states in the dissociation process. In our calculations we used potential curves for various electronic states of different molecular ions of $\mathrm{N}_{2}, \mathrm{O}_{2}$, and $\mathrm{CO}$ that could be found in the literature. By looking at dissociation limits, shapes and slopes of the potential curves and comparing them to the behavior of experimental KER spectra as a function of time, we selected some of them as trial curves for our calculations. 

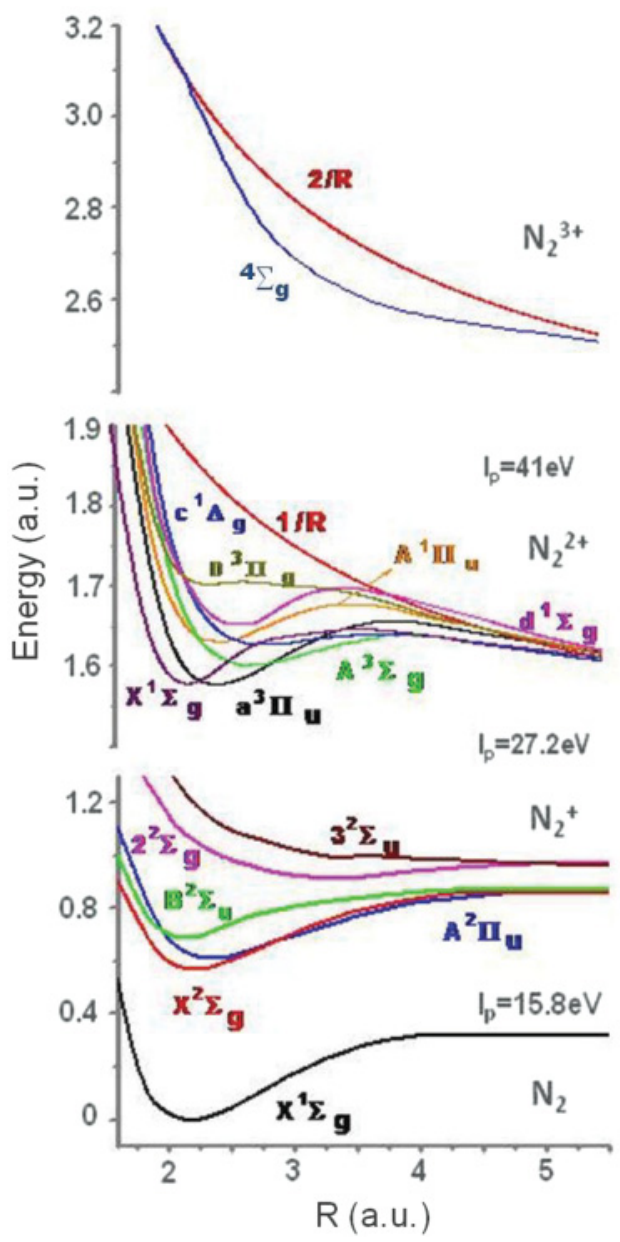

mechanical (QM) point of view the solution to our problem can be found by solving the time-dependent Schrödinger equation (TDSE) with a certain potential. Such an approach accounts for such purely quantum effects as tunneling through a barrier and the initial width and shape and subsequent spread of a nuclear wave packet during its evolution [21]. We used the same Born-Oppenheimer potential energy curves as in the classical simulations. In our QM model the laser pulse is assumed to be short and does not couple the potential curves. After removal of one or two electrons from the neutral molecule at time $\mathrm{t}_{0}=0$ the initial ground state nuclear wave packet was assumed to undergo a Franck-Condon transition to one of the ionic potential energy curves. The field-free propagation on those curves was performed by solving the TDSE on a numerical grid using the Crank-Nicholson scheme [22-24]. After some delay, the probe pulse removes additional electrons, transferring the wave packet onto the final (Coulombic) curve. As for the classical model, in order to take into account the fact that these electrons are not removed instantaneously by the probe pulse of a finite width, we let the wave packet evolve briefly (field-free) on an intermediate state. The time of propagation on that state is a parameter in our model, which we vary to match the calculated zero-delay KERs with the experimental values. As was the case with the classical simulation, we found the optimal time in all cases to be about 5 fs. Finally, to calculate the time-dependent KER spectra, we projected the nuclear wave packets calculated for each time delay onto the continuous Coulomb radial $(l=0)$ wave functions, which are the eigenfunctions of the final repulsive Coulomb potential. The details of the calculation are presented in Appendix B.

\section{RESULTS AND DISCUSSION}

for the $\mathrm{N}_{2}$ molecule and $\mathrm{N}_{2}^{+}, \mathrm{N}_{2}^{2+}$, and $\mathrm{N}_{2}^{3+}$ molecular ions $[19,20]$.

In the experiment several consecutive ionizations can occur during the (pump or probe) pulse with some short time delay, which is a fraction of the pulse duration. The nuclear wave packet (corresponding to a classical particle in this model) can move on one or two additional intermediate states before the Coulomb explosion. The time between these ionization steps allows for a slight change in internuclear distance, which reduces or increases the final KER, depending on the particle's direction of motion. This is reflected by the fact that the experimental KER is always lower than what the model with instantaneous ionization would predict. This effect was accounted for in our calculations by adding one more intermediate state and letting the classical particle move in an average potential for a short time, which we introduce as a parameter. Varying this parameter we matched the calculated energy at zero delay with the experimental value. We reach the best agreement with the experiment when this parameter is equal to $5 \mathrm{fs}$ which is about two thirds of the pulse duration (FWHM). The details of the classical calculations are given in Appendix A.

\section{B. Quantum mechanical model}

A more accurate way of modeling the nuclear dynamic is to take into account quantum effects. From the quantum

\section{A. Nitrogen}

\section{Experimental results}

Figure 3 shows a PIPICO (photoion-photoion coincidence) spectrum where each point represents one pair of ion fragments, with $X$ and $Y$ values representing the two corresponding times of flight. The sharp lines in panel (a) correspond to the real coincidences (with near-zero total momentum) for each breakup channel observed in the experiment, except those with unobserved neutral partners. The rest of the features are due to random coincidences of ions originating from different molecules. To remove the false coincidences while emphasizing the real ones we applied a method of random coincidences subtraction. The method involves subtraction of a similar PIPICO spectrum generated by using pairs of ions produced by two consecutive laser pulses which cannot come from the same molecule. The method is presented in detail in [25]. A subtracted spectrum is shown in Fig. 3(b) from which one can easily identify all breakup channels that we observe in the experiment.

The time-dependent KER spectra for four breakup channels, $\mathrm{N}^{+}+\mathrm{N}^{+}(1,1), \mathrm{N}^{2+}+\mathrm{N}^{+}(2,1), \mathrm{N}^{2+}+\mathrm{N}^{2+}(2,2)$, and $\mathrm{N}^{3+}+\mathrm{N}^{2+}(3,2)$, are presented in Fig. 4. Zero on the delay axis of these plots means that the pump and probe pulses come at the same time. Negative delays correspond to the reverse order of the pulses. If the pump and probe pulses 

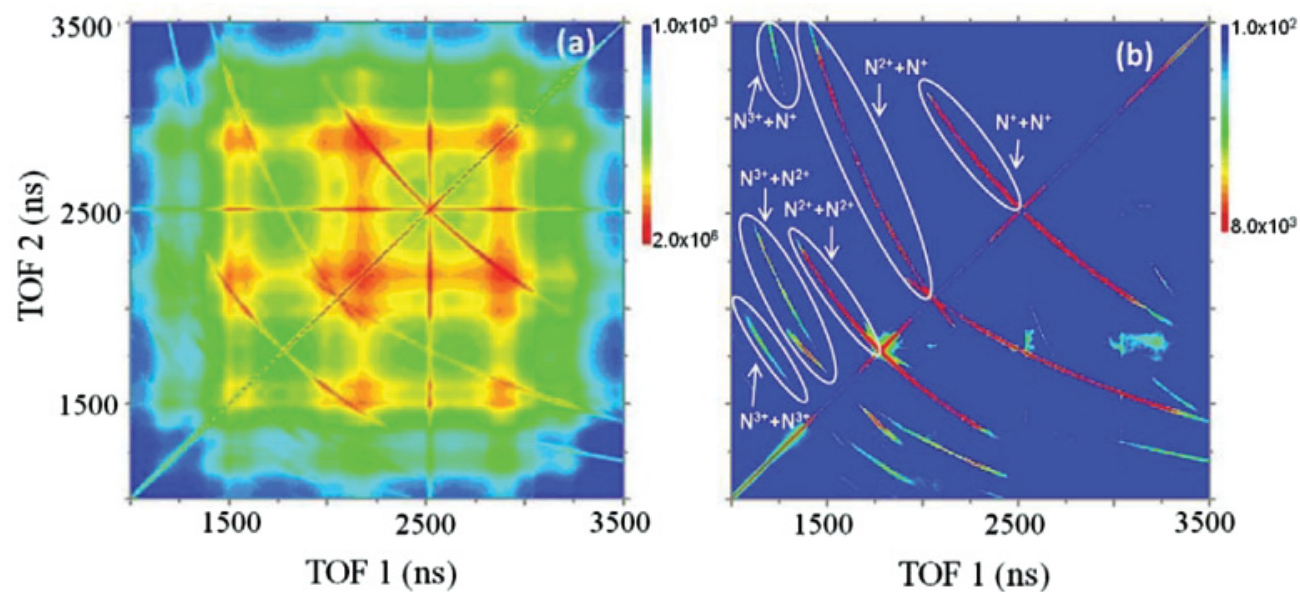

FIG. 3. (Color online) PIPICO spectra for the Coulomb explosion of $\mathrm{N}_{2}$ : vertical and horizontal axes are the times of flight of the first and second ion from each pair of detected ions. (a) The sharp lines are the real coincidences, and the more diffuse background corresponds to random coincidences of ion pairs, originating from different molecules. (b) The same spectrum after subtracting a spectrum of random coincidences generated using pairs of ions from two different laser pulses.

were of the same peak intensity and had the same beam size, this picture would be symmetric about the zero delay. These spectra reveal several time-dependent features that reflect the behavior of parts of the nuclear wave packet, such as its motion on dissociative and quasibound electronic potentials of several transient molecular ions. The descending traces start at about $25 \mathrm{eV}$ for the $(2,1)$ channel, $45 \mathrm{eV}$ for $(2,2)$, and $65 \mathrm{eV}$ for $(3,2)$, and smoothly go down to be separated into distinct traces at larger delays. Each of those traces reaches a certain asymptotic energy, which corresponds to the ultimate energy of molecular dissociation of some intermediate molecular ion. At large delays the probe pulse ionizes ion fragments which are already well separated spatially, so that the second ionization does not add any extra Coulomb energy to the final KER; all the kinetic energy is gained in the intermediate charge state. That allows us to identify intermediate charge states by comparing the asymptotic energies in the time-dependent KER spectra with the single-pulse spectra of various dissociation channels.
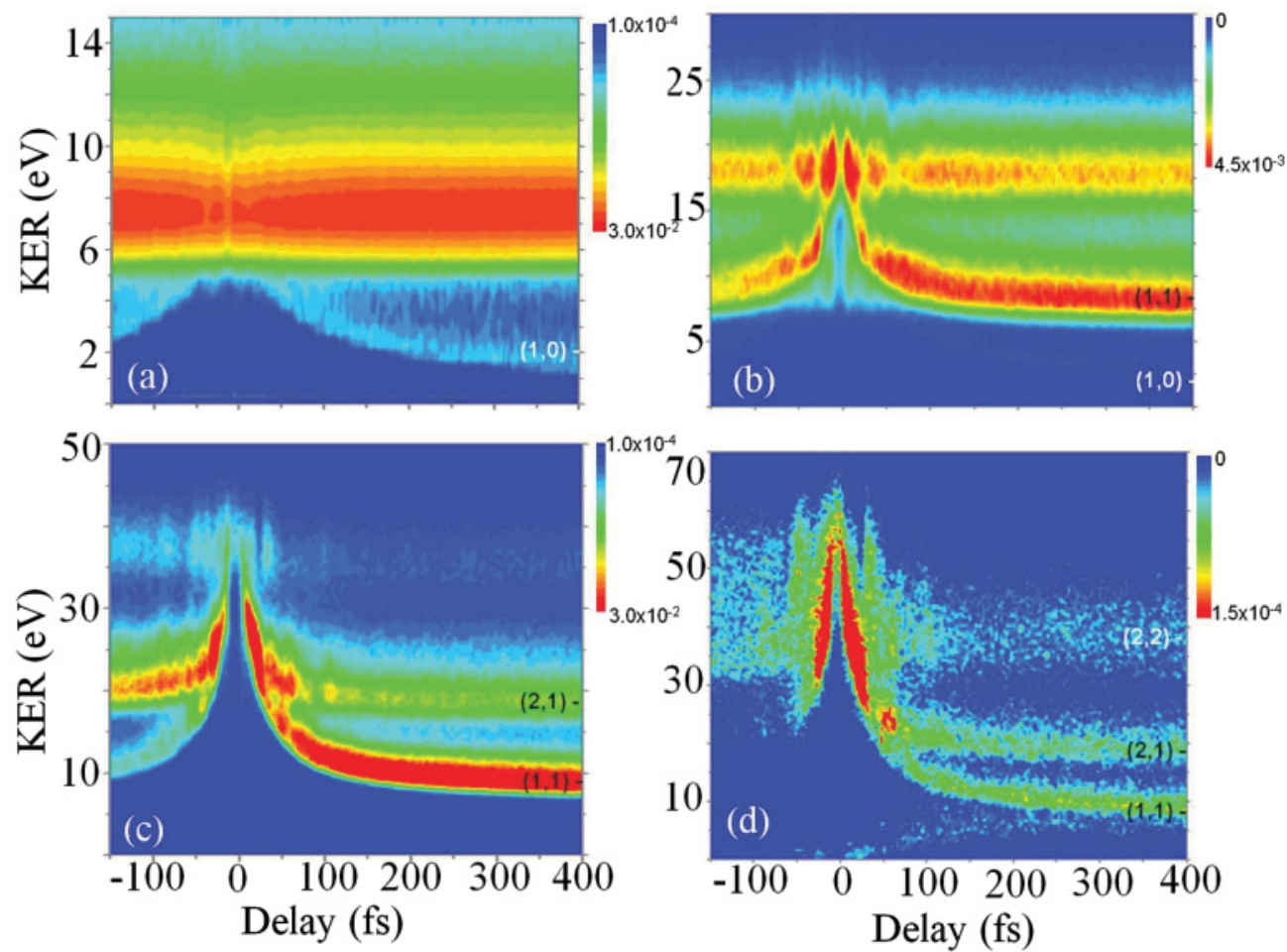

FIG. 4. (Color online) Time-dependent KER spectra (integrated over the solid $4 \pi$ angle) for $\mathrm{N}_{2}$ for (a) $\mathrm{N}^{+}+\mathrm{N}^{+}$, (b) $\mathrm{N}^{2+}+\mathrm{N}^{+}$, (c) $\mathrm{N}^{2+}+\mathrm{N}^{2+}$, and (d) $\mathrm{N}^{3+}+\mathrm{N}^{2+}$ breakup channels. The pump (probe) pulse has a width of 8 fs ( 8 fs) (FWHM) and an intensity of $8 \times 10^{14} \mathrm{~W} / \mathrm{cm}^{2}\left(17 \times 10^{14} \mathrm{~W} / \mathrm{cm}^{2}\right)$. 

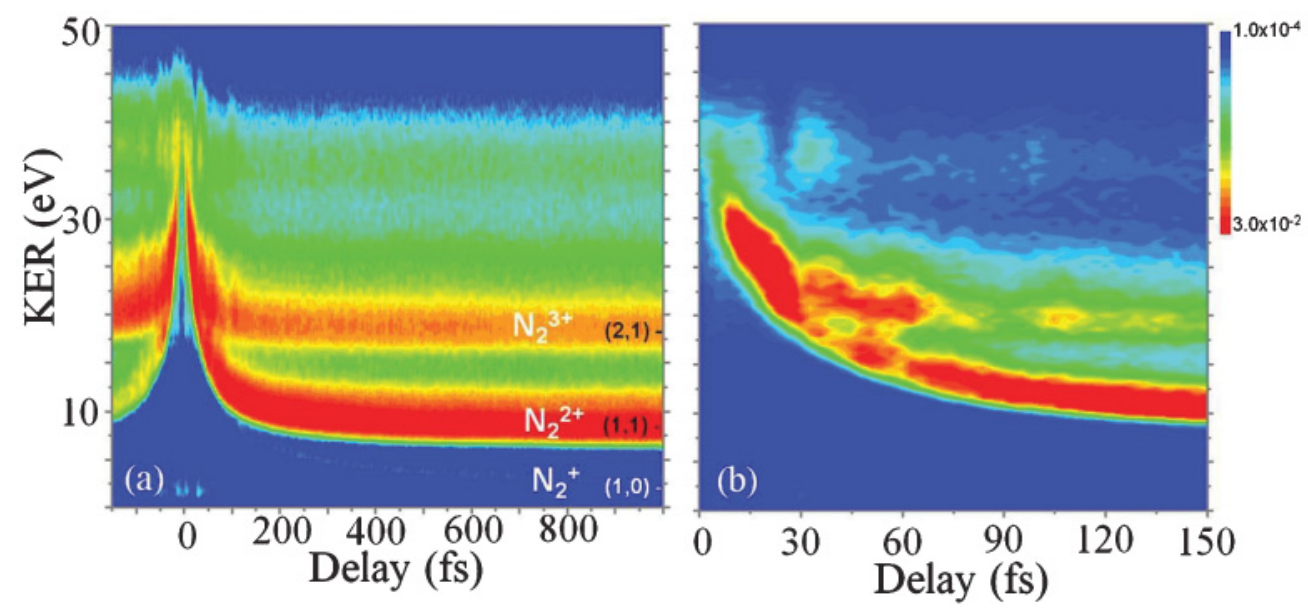

FIG. 5. (Color online) Time-dependent KER spectra (integrated over the full $4 \pi$ solid angle) for $\mathrm{N}^{2+}+\mathrm{N}^{2+}$ breakup channel. The pump (probe) pulse has a width of $8 \mathrm{fs}(8 \mathrm{fs})(\mathrm{FWHM})$ and an intensity of $8 \times 10^{14} \mathrm{~W} / \mathrm{cm}^{2}\left(17 \times 10^{14} \mathrm{~W} / \mathrm{cm}^{2}\right)$. (a) Full spectrum with intermediate charge states indicated. (b) Same spectrum zoomed on the short delay times.

For instance, for the $(2,2)$ final channel (shown in more detail in Fig. 5) there are four possible intermediate dissociative charge states: $(1,0),(1,1),(2,0)$, and $(2,1)$. However, only three dissociative traces are visible in the time-dependent KER spectra. We identify them as originating from $(1,0),(1,1)$, and $(2,1)$ intermediate dissociative channels. The assignment for $(1,1)$ and $(2,1)$ channels is based on the fact that their asymptotic energies at large delays equal peak energies in the KER spectra of the same channels measured in single-pulse experiments. While we cannot use a similar comparison to assign the $(1,0)$ channel, since we do not detect neutral fragments, we can make this assignment by observing that its asymptotic energy is the same as the asymptotic energy of the lone dissociative trace in the time-dependent KER spectra for the $(1,1)$ channel [Fig. 4(a)]. For $(1,1)$ the only possible intermediate dissociative state is $(1,0)$. The time-independent part of the $(1,1)$ spectrum is due to dissociation caused in unexcited molecules by the probe (or pump) pulses alone. Its spectrum is the same as measured for this channel in the single-pulse experiments. The $(1,0)$ channel, together with
$(1,1)$, is also clearly present in the spectra for the $(2,1)$ [Fig. 4(b)], since the only other possible intermediate state in this case is the low probability $(2,0)$.

Similarly, for the $(3,2)$ final channel [Fig. 4(d)], out of seven possible intermediate states- $(1,0),(1,1),(2,0),(2,1)$, $(3,0),(2,2)$, and $(3,1)$ - only three clear dissociative traces are present, and those can be assigned via their asymptotic energies to $(1,1),(2,1)$, and $(2,2)$ channels. The missing intermediate channels are either not produced efficiently by the pump or not ionized efficiently by the probe to yield the final $(3,2)$ state. In general, the relative yield of each channel is determined by the product of relative yields for the first (pump) and the second (probe) ionizations. Clearly, the overall yields are affected by the order of the two nonequivalent pulses, resulting in the time-dependent KER spectra not being symmetric around the zero delay time.

The dependence of the relative yields of different channels on pump and probe pulse parameters is particularly evident in Fig. 6, which presents two more sets of data for the same $(2,2)$ final channel (see caption for details). Despite the
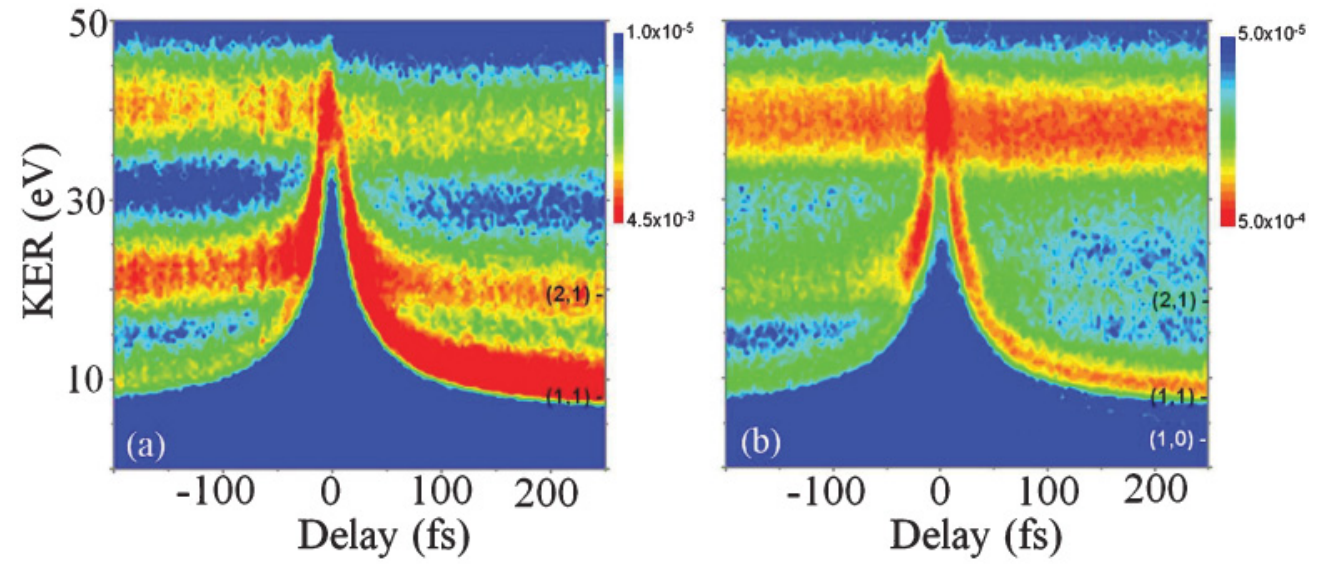

FIG. 6. (Color online) Time-dependent KER spectra (integrated over the full $4 \pi$ solid angle) for the $\mathrm{N}^{2+}+\mathrm{N}^{2+}$ channel. (a) For a pump (probe) pulse duration of $8 \mathrm{fs}(8 \mathrm{fs})$ and intensity of $2 \times 10^{15} \mathrm{~W} / \mathrm{cm}^{2}\left(1.6 \times 10^{15} \mathrm{~W} / \mathrm{cm}^{2}\right)$. (b) For a pump (probe) pulse duration of $8 \mathrm{fs}(8 \mathrm{fs})$ and intensity of $1.6 \times 10^{15} \mathrm{~W} / \mathrm{cm}^{2}\left(1.2 \times 10^{15} \mathrm{~W} / \mathrm{cm}^{2}\right)$. 
obvious differences, the same general features are present in all spectra. While the asymptotic energies of the dissociative traces do not depend on pulse parameters, the high-energy delay-independent part of the spectra does exhibit a noticeable energy shift for different pulse intensities. That part originates from the nondissociative (bound or quasibound) electronic states of the intermediate $\mathrm{N}_{2}^{+}$and $\mathrm{N}_{2}^{2+}$ ions, which are also populated by the pump pulse. The explosion of neutral molecules by the probe (or pump) pulse alone will also result in a similar KER.

That intensity-dependent energy shift can be attributed to different relative contributions to this energy region from $\mathrm{N}_{2}, \mathrm{~N}_{2}^{+}$, and $\mathrm{N}_{2}^{2+}$, each resulting in slightly different (but overlapping) KER spectra.

It is also interesting to note that the clear presence of the $(1,0)$ dissociative traces in the $(1,1),(2,1)$, and $(2,2)$ final channels indicates that tunneling single ionization of $\mathrm{N}_{2}$ by intense few-cycle near-IR laser pulses produces electronically excited $\mathrm{N}_{2}^{+*}$ ions which can dissociate (the ground state cannot, see Fig. 2) with substantial probability. This follows from the fact that the molecular ion's ground and lowest excited states are not dissociative (Fig. 2). The possible mechanisms for this excitation include recollision [26-28], resonant excitation, [29] and shake-up [30].

The high-energy (bound-state) part of time-dependent KER spectra exhibits a time-dependent structure indicative of coherent vibrational motion taking place in bonding electronic states of $\mathrm{N}_{2}^{+}$and $\mathrm{N}_{2}^{2+}$. Careful measurement and analysis of that vibrational motion could, in principle, reveal exactly which electronic states are populated by the pump. While low statistics and a rather narrow range of delays in our coincidence experiments precluded us from conducting such analysis here, in another recent study of $\mathrm{O}_{2}$ performed with a velocity map imaging apparatus [31] we were able to observe both vibrational structure and vibrational revivals and to identify the active electronic states based on the measured revival times.

\section{Comparison with classical simulations}

While the intermediate dissociation channels are straightforwardly identified by their fragment charges via the asymptotic energies, each of those channels can proceed through a number of different electronic states having the same dissociation limit. It is sometimes possible to identify different electronic states by their distinct energies in high-resolution KER spectra [13]. Can specific dissociative electronic states be assigned based on our time-dependent KER spectra? To answer that question we performed a series of classical and quantum mechanical simulations described in Sec. III and Appendixes A and B. In the following discussion we focus on the $(2,2)$ final channel.

The results of the classical simulations for the final $(2,2)$ channel are presented in Fig. 7. By plotting the calculated curves on top of the experimental spectrum, we can immediately see that even this simple model gives us good insight into the fragmentation process. Both asymptotic energies and overall shapes of dissociative traces are faithfully reproduced by the simulations.

For the $\mathrm{N}_{2}^{+}$intermediate ion, out of two dissociative states used in our calculations $\left(2^{2} \Sigma_{g}\right.$ and $\left.3^{2} \Sigma_{u}\right)$ only one $\left(2^{2} \Sigma_{g}\right)$ fit

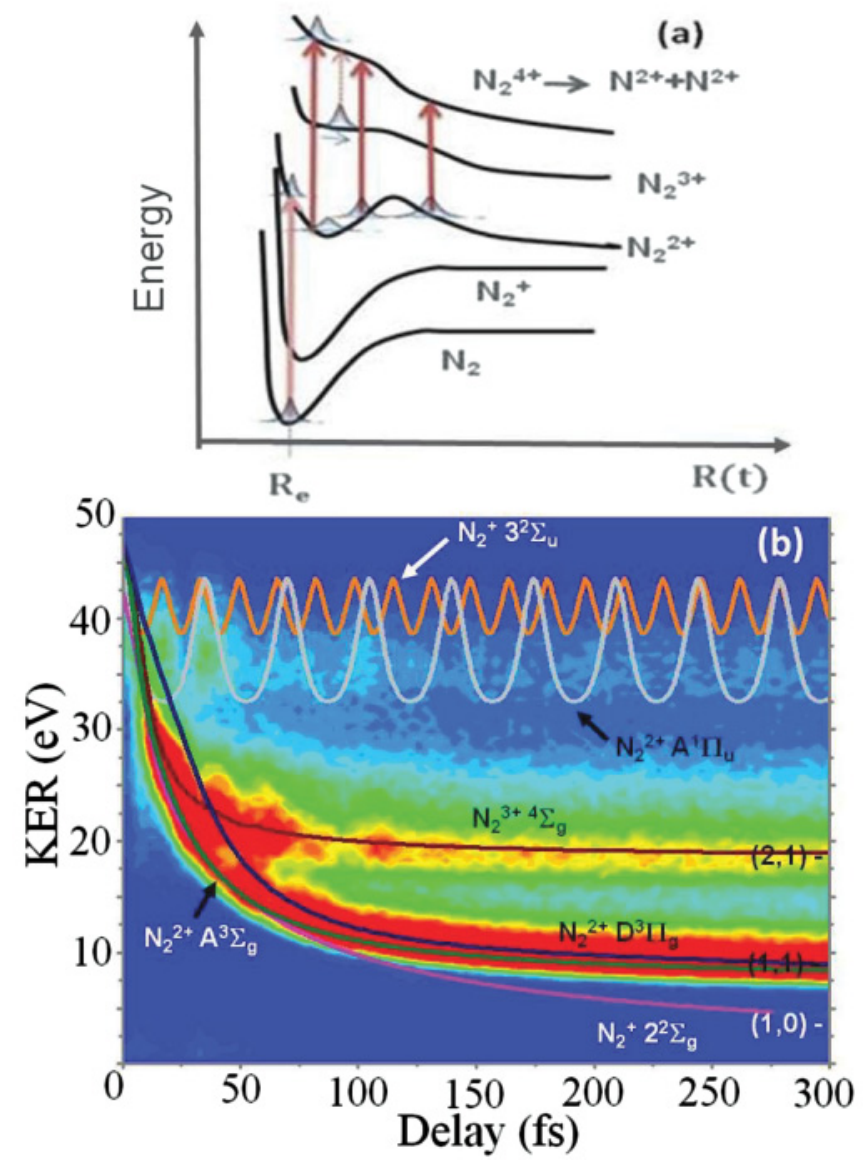

FIG. 7. (Color online) Classical model results for the $\mathrm{N}^{2+}+\mathrm{N}^{2+}$ breakup channel. (a) Schematic presentation of the wave-packet motion on potential energy curves. (b) Classically calculated timedependent KER traces superimposed on the experimental spectrum. Each line corresponds to a certain intermediate electronic state of the molecular ion as indicated. The laser parameters are the same as in Fig. 5.

the experimental trace. The experimental dissociation trace, which corresponds to the $\mathrm{N}_{2}^{2+}$ intermediate ion, is broad, indicating that several electronic states of the ion may be contributing to it. This is not surprising, since the density of those $\mathrm{N}_{2}^{2+}$ electronic states that dissociate directly after being populated in the Frank-Condon region is high (see Fig. 2). Three of the four $\mathrm{N}_{2}^{2+}$ potential energy curves we tried $\left(c^{1} \Delta_{g}, A^{3} \Sigma_{g}\right.$, and $\left.A^{1} \Pi_{u}\right)$ fit well within the experimental trace, while only one $\left(D^{3} \Pi_{g}\right)$ deviated from it enough to be clearly excluded. For the $\mathrm{N}_{2}^{3+}$ ion we only tried one potential energy curve of several found in the literature $\left({ }^{4} \Sigma_{g}\right)$ [21], which was the most divergent from the Coulombic $(2 / R)$ potential. The corresponding classical result fits the experimental trace very well. All non-Coulombic states of $\mathrm{N}_{2}^{3+}$ calculated in [21] are dissociative and very close to each other in energies and shapes, having the same dissociation limit.

The analysis is less obvious for the oscillating (bound) part of the spectra in Fig. 7(b). Both $\mathrm{N}_{2}^{+}$and $\mathrm{N}_{2}^{2+}$ have a number of (quasi-)bound potential energy curves that would result in overlapping KER spectra. The structure that we see at short delays cannot be exactly matched with classical oscillations in any of the potential wells in Fig. 2. However, not being able to 

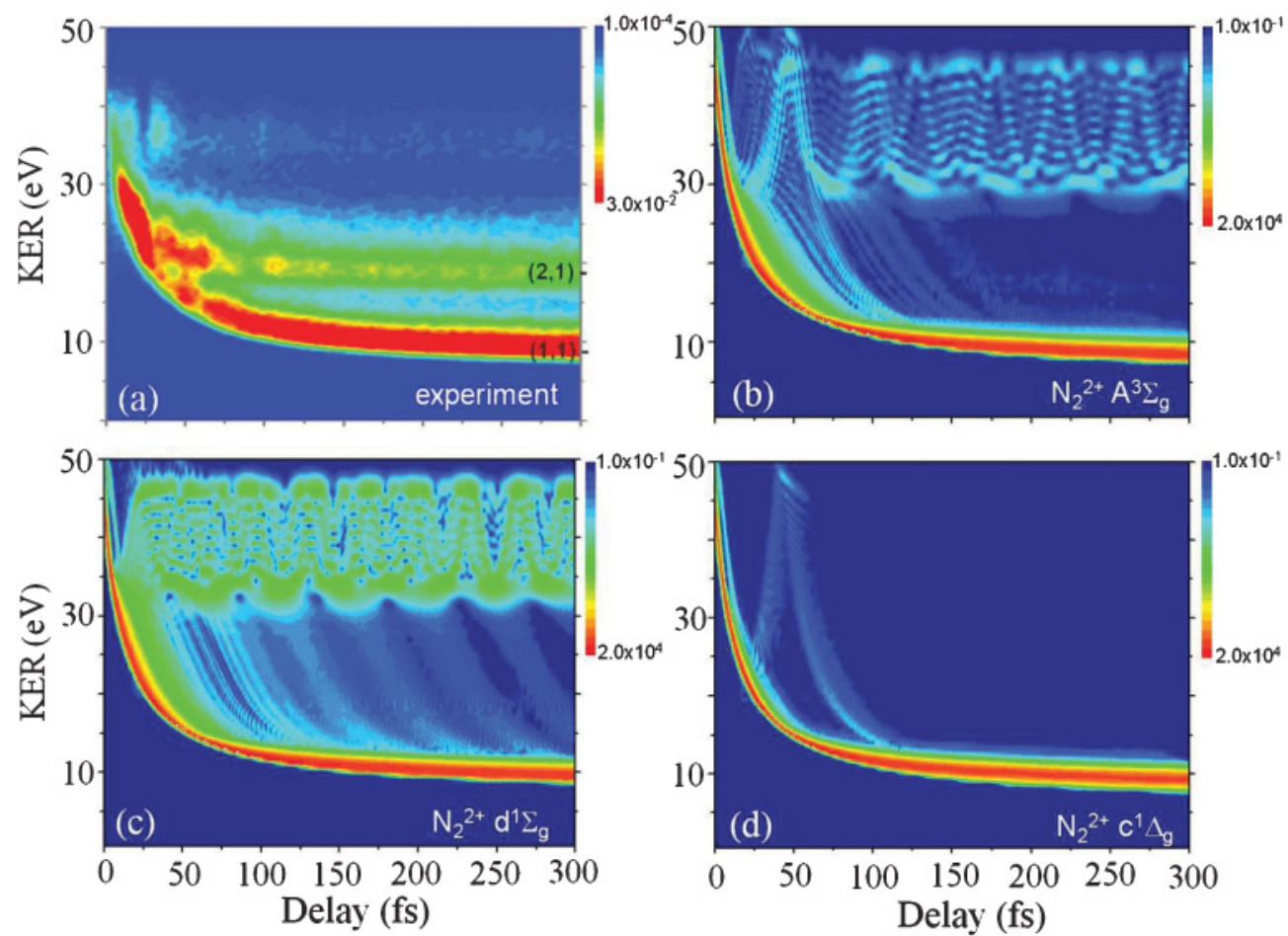

FIG. 8. (Color online) Simulated time-dependent KER spectra for various possible dissociation pathways. (a) The experimental spectrum is shown for comparison. (b) $\mathrm{N}_{2} \rightarrow \mathrm{N}_{2}^{2+}\left(A^{3} \Sigma_{g}\right) \rightarrow \mathrm{N}_{2}^{4+} \rightarrow \mathrm{N}^{2+}+\mathrm{N}^{2+}$. (c) $\mathrm{N}_{2} \rightarrow \mathrm{N}_{2}^{2+}\left(d^{1} \Sigma_{g}\right) \rightarrow \mathrm{N}_{2}^{4+} \rightarrow \mathrm{N}^{2+}+\mathrm{N}^{2+}$. (d) $\mathrm{N}_{2} \rightarrow \mathrm{N}_{2}^{2+}\left(c^{1} \Delta_{g}\right) \rightarrow$ $\mathrm{N}_{2}^{4+} \rightarrow \mathrm{N}^{2+}+\mathrm{N}^{2+}$. The laser parameters are the same as in Fig. 5.

resolve the oscillation frequencies, we still can rule out those states that result in KER too high or too low in comparison to the experimental values. For instance, we can rule out the $A^{2} \Pi_{u}$ state of $\mathrm{N}_{2}^{+}$, while the $A^{1} \Pi_{u}$ state of $\mathrm{N}_{2}^{2+}$ is a clear possibility [cf. Fig. 7(b)].

\section{Comparison with quantum mechanical simulations}

A classical simulation cannot capture some important features of quantum wave-packet motion, such as tunneling, over-barrier reflection or spread of the wave packets. To see how important the quantum effects are, we also performed quantum mechanical simulations, which generated the calculated time-dependent KER spectra shown for the final $(2,2)$ channel in Figs. 8-11. Panels (b), (c), and (d) of Fig. 8 show the calculated spectra for wave-packet evolution on three different electronic states of $\mathrm{N}_{2}^{2+}: A^{3} \Sigma_{g}, d^{1} \Sigma_{g}$, and $c^{1} \Delta_{g}$. At the starting point of the evolution, a wave function is centered at 2.2 a.u. (Franck-Condon transition from $\mathrm{N}_{2} X^{1} \Sigma_{g}$ ). For the $A^{3} \Sigma_{g}$ and $d^{1} \Sigma_{g}$ the largest part of the wave packet goes over the potential barrier and dissociates, but a small part is trapped in a shallow potential well and keeps oscillating. We also observe weak dissociation traces going down to the same asymptotic energy after each oscillation, indicating tunneling through the potential barrier. The $c^{1} \Delta_{g}$ state seems to not have any vibrational states but has a very shallow depth, which forces a part of the wave packet to bounce once and then to completely dissociate. Asymptotic energies for all these states converge to the experimental value.

There are three more calculated KER spectra for the $\mathrm{N}_{2}^{2+}$ states shown in Fig. 9: $a^{3} \Pi_{u}, A^{1} \Pi_{u}$, and $D^{3} \Pi_{g}$. The $a^{3} \Pi_{u}$ and $A^{1} \Pi_{u}$ states both have prominent bound parts with large amplitude and high energies compared to the experimental spectra. The $D^{3} \Pi_{g}$ state has a small feature which looks like a reflection in a shallow well of the potential.

Figure 10 presents an excellent agreement between the calculated spectrum for the wave-packet evolution on the ${ }^{2} \Sigma_{g}^{+}$ dissociative state of $\mathrm{N}_{2}^{+}$and the weak dissociative trace in the experimental spectrum. Most dissociative states of the $\mathrm{N}_{2}^{+}$ion are highly excited states [20], which cannot be easily populated at our experimental conditions. The lowest energy states are bound. Taking any of them as a trial potential in either classical or quantum model calculations will result in pure oscillatory spectra with energies that are much higher than those measured in our experiments.

Figure 11 reveals the origin of the dissociative feature with high asymptotic energy. A wave packet launched on the dissociative non-Coulombic ${ }^{4} \Sigma_{g}$ state of the $\mathrm{N}_{2}^{3+}$ ion follows the experimental trace in the KER spectrum. Among the electronic states of the $\mathrm{N}_{2}^{3+}$ ion with the lowest dissociation limit, ${ }^{4} \Sigma_{g}$ was picked as the most diverging from the Coulomb $2 / R$ potential according to [21] (cf., Fig. 2). Judging from the width and intensity of the corresponding trace in Fig. 11(a), the intensity of the pump pulse is sufficient to populate all the states of $\mathrm{N}_{2}^{3+}$ that converge to the lowest dissociation limit.

\section{Asymmetric breakup and electron localization}

In addition to the dissociation channels already discussed, we also measured a time-dependent KER spectrum for the asymmetric $(3,1)$ channel: $\mathrm{N}_{2}^{4+} \rightarrow \mathrm{N}^{3+}+\mathrm{N}^{+}$. This spectrum, presented in Fig. 12, reveals a very peculiar behavior of 

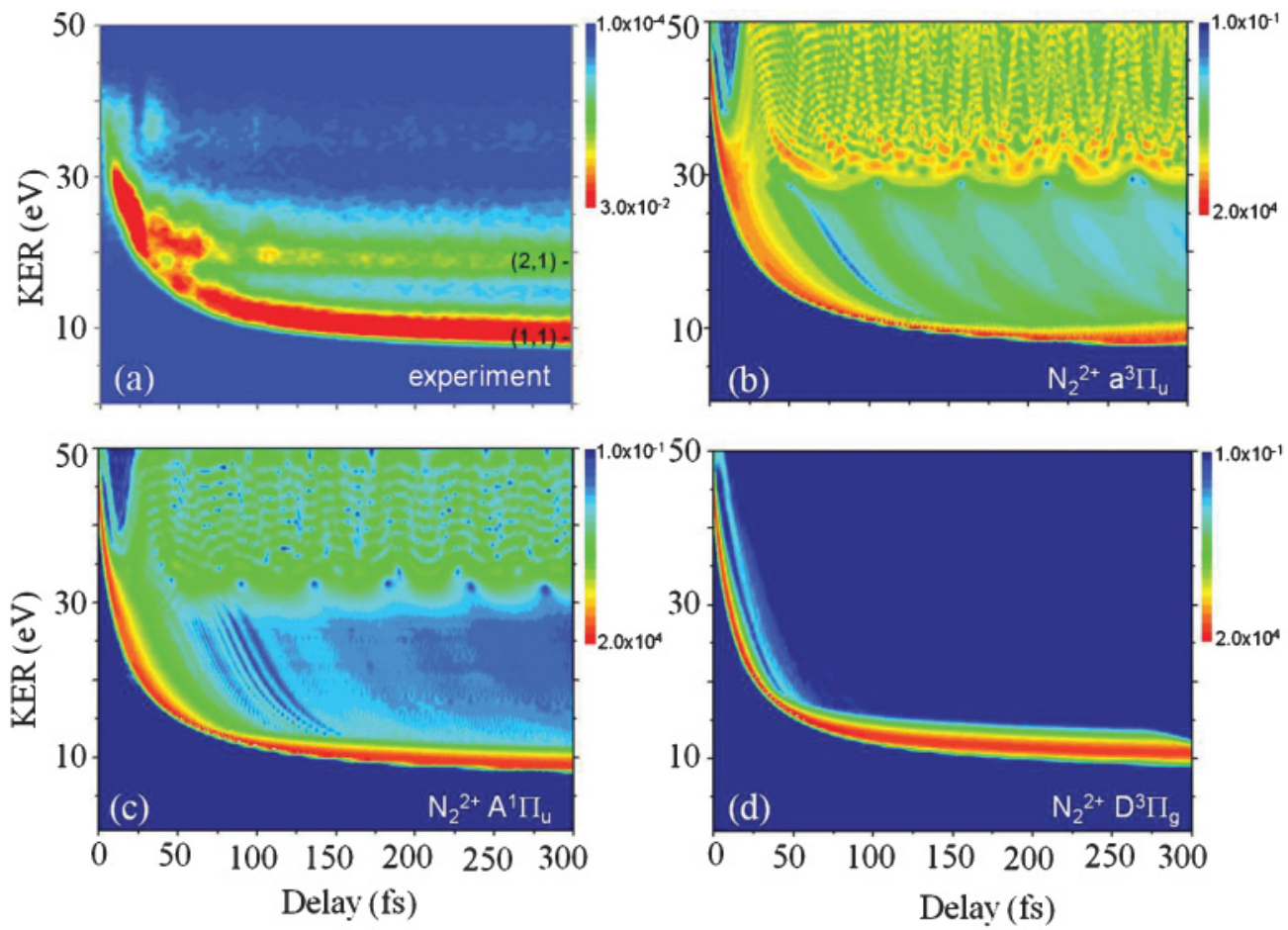

FIG. 9. (Color online) Simulated time-dependent KER spectra for various possible dissociation pathways. (a) The experimental spectrum is shown for comparison; (b) $\mathrm{N}_{2} \rightarrow \mathrm{N}_{2}^{2+}\left(a^{3} \Pi_{u}\right) \rightarrow \mathrm{N}_{2}^{4+} \rightarrow \mathrm{N}^{2+}+\mathrm{N}^{2+}$. (c) $\mathrm{N}_{2} \rightarrow \mathrm{N}_{2}^{2+}\left(A^{1} \Pi_{u}\right) \rightarrow \mathrm{N}_{2}^{4+} \rightarrow \mathrm{N}^{2+}+\mathrm{N}^{2+}$. (d) $\mathrm{N}_{2} \rightarrow$ $\mathrm{N}_{2}^{2+}\left(D^{3} \Pi_{g}\right) \rightarrow \mathrm{N}_{2}^{4+} \rightarrow \mathrm{N}^{2+}+\mathrm{N}^{2+}$. The laser parameters are the same as in Fig. 5.

this charge-asymmetric dissociation: the dissociation trace is present only for delays less than $20 \mathrm{fs}$ and disappears completely for longer delays. While it is operational, it behaves similarly to all dissociative traces observed in other channels, starting at around $36 \mathrm{eV}$ at zero delay and going down to about $17 \mathrm{eV}$ at $20 \mathrm{fs}$. What is striking is that the yield of this channel abruptly goes to zero at around $20 \mathrm{fs}$ and remains zero thereafter.

The phenomenon of charge asymmetric dissociation was observed in earlier studies with single pulses longer than 30 fs [12]. That study concluded, based on indirect evidence, that in $\mathrm{N}_{2}$ the $(3,1)$ channel is created only at small internuclear separations early in the pulse. Our time-resolved measurement is the first direct experimental validation of that conclusion. We propose a simple intuitive explanation for this peculiar behavior. The pump pulse triply ionizes neutral $\mathrm{N}_{2}$ and the resulting molecular tri-cation $\mathrm{N}_{2}^{3+}$ starts to dissociate. After the dissociating $\mathrm{N}_{2}^{3+}$ reaches some critical internuclear distance, the unpaired electron becomes localized near one of the two nuclei, yielding a pair of distinct atomic ions $\mathrm{N}^{2+}$ and $\mathrm{N}^{+}$. Once that happens, any further single ionization by the probe pulse will remove the electron with much higher probability from $\mathrm{N}^{+}$(rather than from $\mathrm{N}^{2+}$ ) due to its much lower ionization potential. Therefore, the emerging overwhelming dominance of the symmetric $(2,2)$ channel over the asymmetric $(3,1)$ channel signifies that the unpaired electron is completely localized.
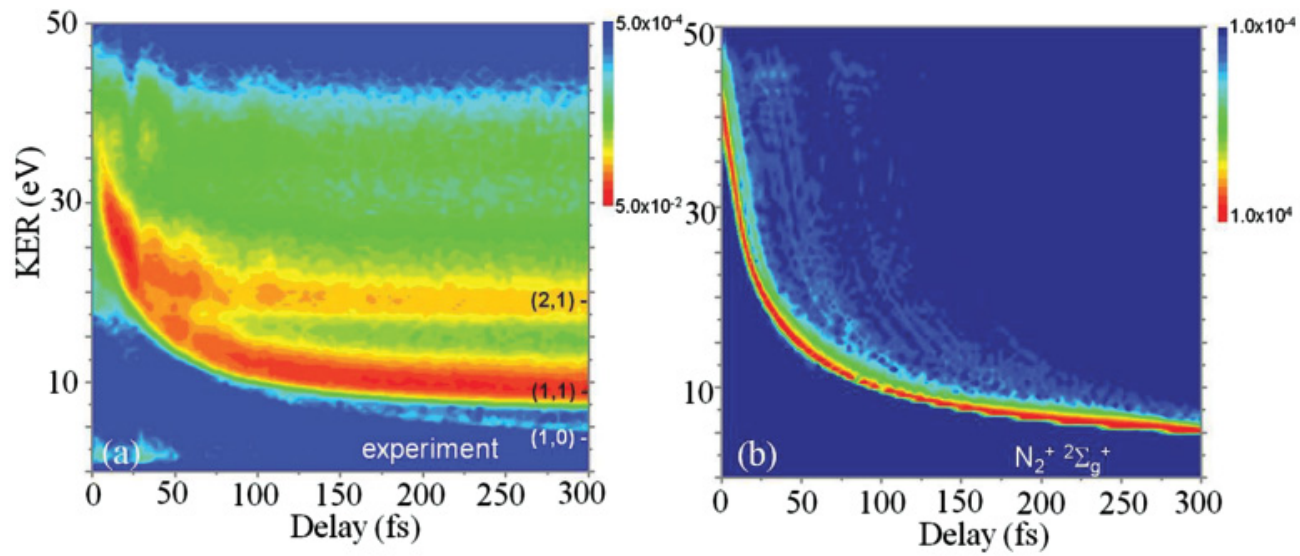

FIG. 10. (Color online) (a) Experimental spectrum and (b) simulated time-dependent KER spectra for $\mathrm{N}_{2} \rightarrow \mathrm{N}_{2}^{+}\left({ }^{2} \Sigma_{g}^{+}\right) \rightarrow \mathrm{N}_{2}^{4+} \rightarrow$ $\mathrm{N}^{2+}+\mathrm{N}^{2+}$ dissociation pathway. The laser parameters are the same as in Fig. 5. 

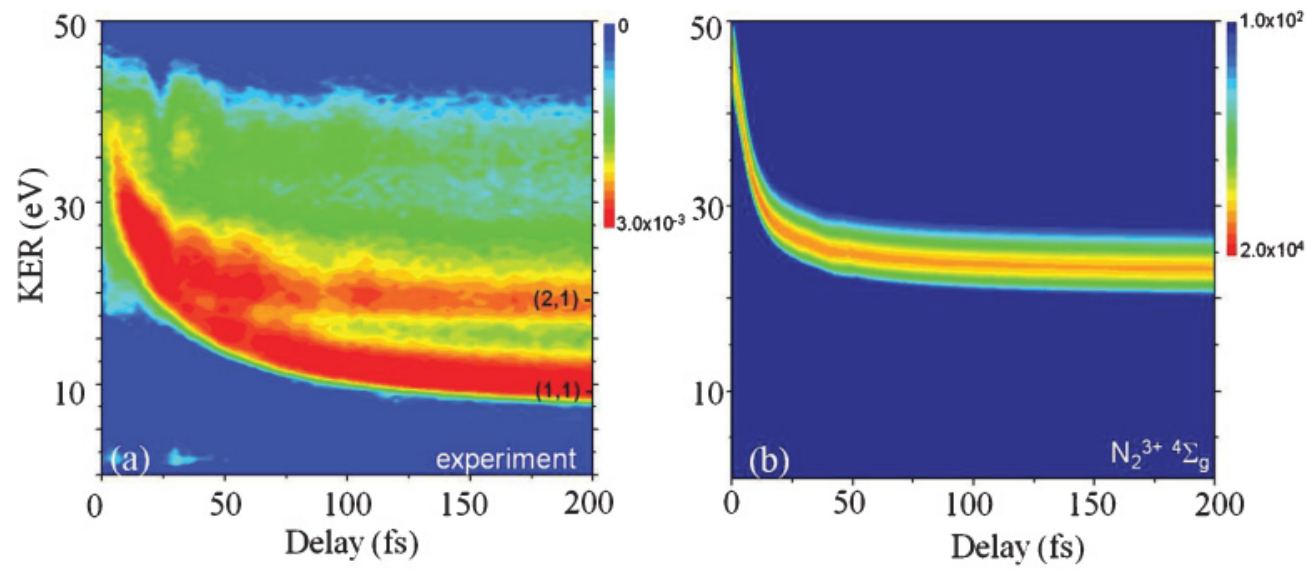

FIG. 11. (Color online) (a) Experimental spectrum and (b) simulated time-dependent KER spectra for the $\mathrm{N}_{2} \rightarrow \mathrm{N}_{2}^{3+}\left({ }^{4} \Sigma_{g}\right) \rightarrow \mathrm{N}_{2}^{4+} \rightarrow$ $\mathrm{N}^{2+}+\mathrm{N}^{2+}$ dissociation pathway. The laser parameters are the same as in Fig. 5.

Such electron localization was postulated to play a crucial role in enhanced tunneling ionization at the critical distance of homonuclear diatomic molecules [10]. While unambiguously supported by theoretical modeling only in $\mathrm{H}_{2}^{+}[5,10]$, this phenomenon [often referred to also as charge-resonance enhanced ionization (CREI) [5] is believed to be universal, at least for diatomic molecules, with the critical distance estimated to be also universally around 2-3 times the equilibrium bond length for the neutral molecule. From our time-dependent KER spectra we can easily estimate the internuclear distance at which the electron localization occurs to be about 2.5 times the bond length for neutral molecules in their ground electronic state. It would be interesting to resolve the detailed temporal dynamics of such electron localization, but that study will likely require much shorter (attosecond) pulses.

\section{B. Oxygen}

The time-dependent KER spectra for four breakup channels $\mathrm{O}^{+}+\mathrm{O}^{+}(1,1), \mathrm{O}^{2+}+\mathrm{O}^{+}(2,1), \mathrm{O}^{2+}+\mathrm{O}^{2+}(2,2)$, and $\mathrm{O}^{3+}+$ $\mathrm{O}^{+}(3,1)$ are shown in Fig. 13. The main features of these spectra are similar to those seen in nitrogen. For the $(1,1)$ channel there is a dissociative trace that starts to be visible

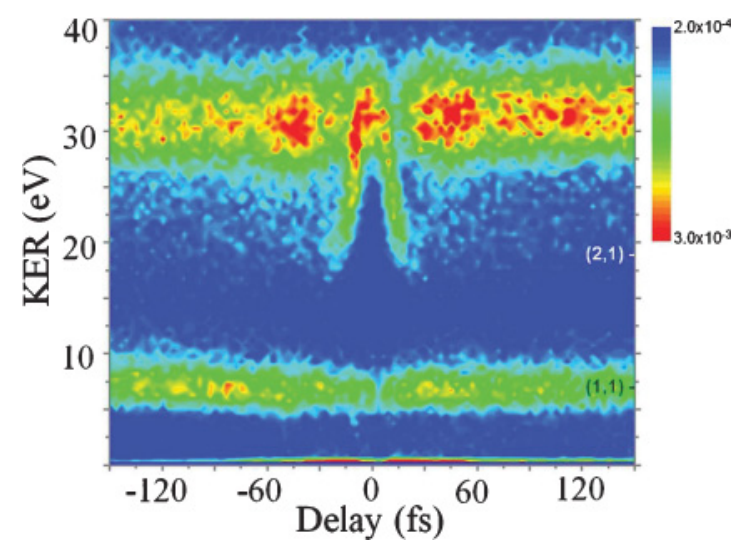

FIG. 12. (Color online) The experimental time-dependent KER spectrum (integrated over the full $4 \pi$ solid angle) for the $\mathrm{N}^{3+}+\mathrm{N}^{+}$ breakup channel. The laser parameters are the same as in Fig. 5. below $6 \mathrm{eV}$ after 40 fs of delay and goes down to below $3 \mathrm{eV}$ at $200 \mathrm{fs}$. The high-energy part of the $(1,1)$ spectrum represents $\mathrm{O}^{+}$fragments that are produced by the probe pulse from the (quasi-)bound states of $\mathrm{O}_{2}^{+}$and also from the neutral $\mathrm{O}_{2}$ molecules that were not ionized by the pump. For the $(2,1)$ and $(2,2)$ channels we observe time-dependent behavior inside the high-energy region. In addition to the common features observed in both $\mathrm{O}_{2}$ and $\mathrm{N}_{2}$ KER spectra, such as dissociation traces which start at zero delay, one can see that for oxygen, parts of the wave packet start their trips down in energy later in time, after being bound in some potential well.

More specifically, we focus on the $(2,2)$ channel [Fig. 13(c)], which is characterized by high statistics and clear dynamics features at the same time. We observed that, unlike in nitrogen, in $\mathrm{O}_{2}$ the population is distributed more evenly between dissociative and bound states. From that observation, one can conclude that bound or quasibound intermediate states of the parent ions are energetically more accessible and more easily populated for oxygen than for nitrogen at our experimental conditions. Two dissociative traces are also almost equally intense and come very close to each other in energy (around 7 and $11 \mathrm{eV}$ at $200 \mathrm{fs}$ ). Those dissociative traces that start at later delays come to merge with the $11 \mathrm{eV}$ trace, suggesting that electronic states of the same parent $\mathrm{O}_{2}^{q+}$ ion are populated and cause this type of dynamics.

For nitrogen, in contrast, the bound part of the spectrum is weak in comparison to the dissociative part. It is also narrower in energy range, reflecting very small displacement of bound potential energy curves upon its ionization. For $\mathrm{N}_{2}$ the dissociation trace starts at zero delay and any leaking of the oscillating wave packet out of the potential well is negligibly small, so it cannot be clearly distinguished in the experimental plots. Hence it can be suggested that bound or quasibound intermediate electronic states are hard to access in our experimental conditions and their potential wells are deeper in nitrogen than in oxygen. Similar to $\mathrm{N}_{2}$, KER spectra for $\mathrm{O}_{2}$ are recorded starting from negative delays. The asymmetry of the spectra about zero time is due to different parameters (peak intensities and beam sizes) of the pump and probe pulses, and it reflects different population distribution and ionization probabilities of the intermediate states. 

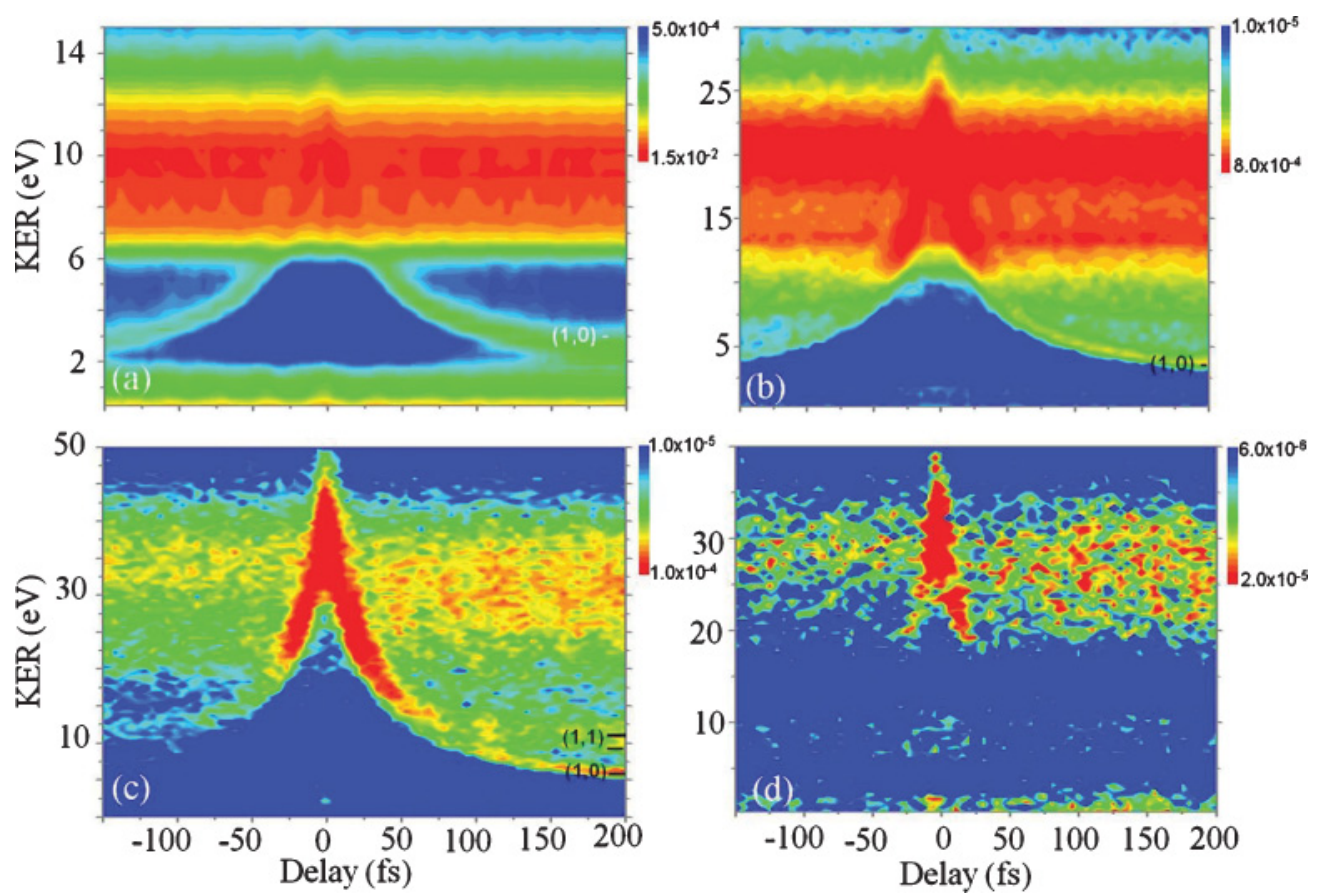

FIG. 13. (Color online) The experimental time-dependent KER spectra (integrated over the solid $4 \pi$ angle) for $\mathrm{O}_{2}$ for $\left(\right.$ a) $\mathrm{O}^{+}+\mathrm{O}^{+}$, (b) $\mathrm{O}^{2+}+\mathrm{O}^{+}$, (c) $\mathrm{O}^{2+}+\mathrm{O}^{2+}$, and (d) $\mathrm{O}^{3+}+\mathrm{O}^{+}$breakup channels. The laser parameters are the same as in Fig. 5.

The asymmetric $(3,1)$ breakup channel, $\mathrm{O}^{3+}+\mathrm{O}^{+}$, was also observed in our experiment (see figure 13(d)). The same phenomenon is observed for oxygen that we noted for the nitrogen asymmetric breakup: the bright dissociation trace that appears at zero delay at around $40 \mathrm{eV}$ goes down to about $18 \mathrm{eV}$ and then disappears completely at 20 fs. This observation adds up to the conclusion that this phenomenon is not specific to nitrogen molecule, but it is rather common for homonuclear diatomics, similarly resulting from localization of an unpaired electron in dissociating $\mathrm{O}_{2}^{3+}$ when its internuclear distance reaches about 2.5 times the neutral molecule's bond length. Such electron localization appears to be a necessary step in the universal mechanism of enhanced ionization of homonuclear diatomic molecules by intense near-IR laser pulses. It must also be universal (under appropriate conditions) for all oddly charged molecular cations, most notably also taking place in singly charged molecular ions. To confirm that hypothesis, one would need to measure the time-dependent yield of the asymmetric $(2,0)$ channel and verify that it also disappears after similarly short delays. Unfortunately, our detection scheme does not allow correlated measurements for channels with neutral fragments, and uncorrelated detection of doubly charged atomic ions cannot be conclusive since those ions are produced via several possible channels.

The next step is to apply our classical and quantum models, which we already tried on the nitrogen molecule, to extract the information about the parent ions and the electronic states contributing to the dynamics observed in the oxygen experiment. Following the classical model described in Sec. III, we simulate the KER for the different breakup channels observed in the experiment. For instance, the kinetic energy release for the $\mathrm{O}^{2+}+\mathrm{O}^{2+}$ channel at a certain time is calculated as a sum of the kinetic energy acquired by the moving nuclei by that time, and the Coulomb-repulsion energy of the ionic fragments at internuclear separation reached by that time. Potential energy curves for oxygen ions $\mathrm{O}_{2}^{q+}$ are presented in Fig. 14 .

The results of our classical calculations and a schematic drawing of the wave-packet motion on the potential energy curves are presented in Fig. 15. The potential energy curves for the neutral molecule and molecular ions were found in Refs. [32-36]. As for the nitrogen molecule, we plot the classically calculated traces on top of the experimental spectra for the $\mathrm{O}^{2+}+\mathrm{O}^{2+}$ channel. As seen in Fig. 15, the upper dissociation trace which converges to an asymptotic energy of about $11 \mathrm{eV}$ after $200 \mathrm{fs}$ can be assigned to some electronic states of the doubly charged molecular ion (only $A^{3} \Sigma_{u}$ is shown in Fig. 15(b) but several other states lead to very similar traces). The lower trace appears to mostly characterize dissociation via population of excited states of the singly charged molecular ion (i.e., ${ }^{4} \Delta_{g}$ ).

Obviously, it is impossible to reproduce quantum effects of the wave-packet motion, such as leaking from the potential well, with a purely classical model. Nonetheless, we tried to model the oscillatory part classically, like we did for the $\mathrm{N}_{2}$ molecule. Though, it did not allow us to assign a particular electronic state, it did allow us to exclude those states which do not reproduce the correct KER range.

To better account for the quantum effects, we used the quantum model described in the second part of Sec. III. As described earlier, the model is based on solving the TDSE for the nuclear wave-packet motion on the potential energy curves of $\mathrm{O}_{2}^{q+}$ ions. The wave packet is launched by the pump pulse by singly or multiply ionizing the neutral oxygen molecule. The KER as a function of delay time for the $(2,2)$ channel was calculated by projecting the wave packet onto 


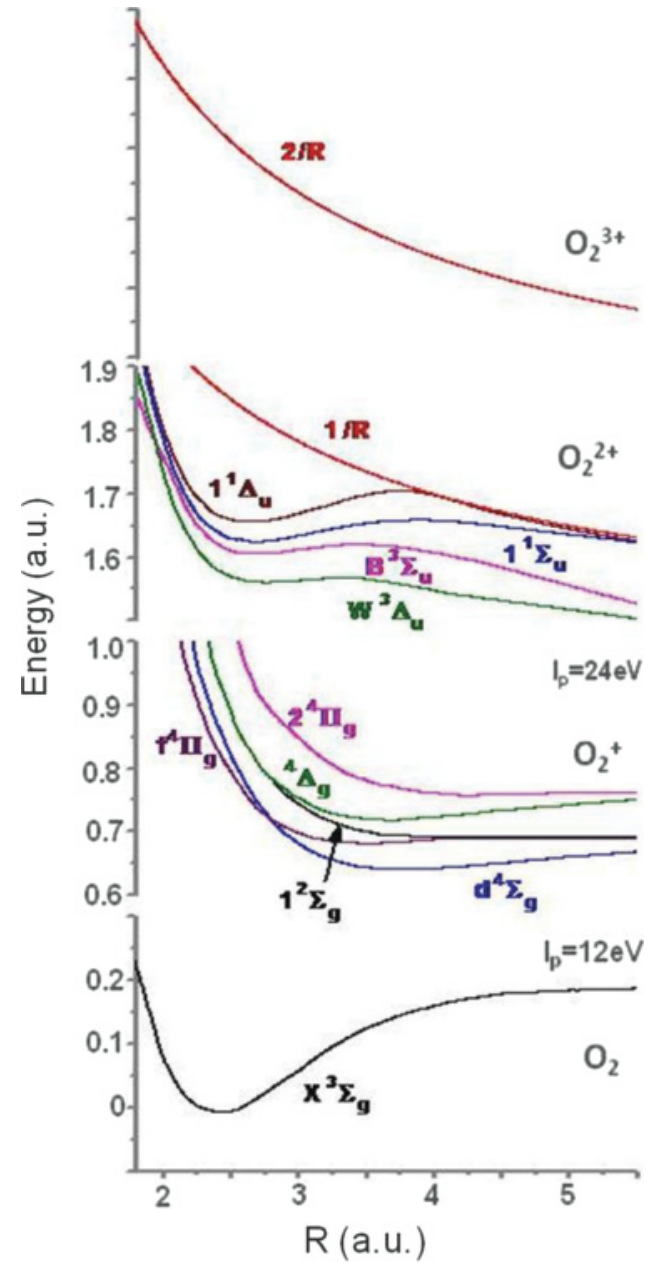

FIG. 14. (Color online) Several low-lying potential energy curves for the $\mathrm{O}_{2}$ molecule and $\mathrm{O}_{2}^{+}, \mathrm{O}_{2}^{2+}, \mathrm{O}_{2}^{3+}$ molecular ions [32-36].

the corresponding Coulombic wave function at each delay. The calculated spectra for the $(2,2)$ channel, together with the experimental spectra, are shown in Figs. 16 and 17.

Panels (b), (c), (d), (e) and (f) of Fig. 16 show the spectra for wave packets evolving on five different electronic states of the $\mathrm{O}_{2}^{+}$ion: $2^{4} \Pi_{g}, 1^{2} \Sigma_{g}, d^{4} \Sigma_{g}, f^{4} \Pi_{g}$, and ${ }^{4} \Delta_{g}$ respectively. At the starting point of the evolution the wave function is centered at 2.4 a.u. (Franck-Condon transition from $\mathrm{O}_{2} X^{3} \Sigma_{g}$ ). All of these states are exhibiting very similar behavior. They are all dissociative with very shallow potential wells which contain a few vibrational states [37]. Therefore, most of the $\mathrm{O}_{2}^{+}$nuclear wave packet dissociates immediately with about the same asymptotic kinetic energy release of 5-7 eV, which is observed in our experiments.

A small part of the wave packet (orders of magnitude smaller: see the color scheme to Fig. 17) stays bound for a while but leaks out after a few oscillations and dissociates to the same KER. Four more spectra for the nuclear wave-packet propagation on $1^{1} \Delta_{u}, B^{3} \Sigma_{u}, W^{3} \Sigma_{u}$, and $1^{1} \Sigma_{u}$ states of $\mathrm{O}_{2}^{2+}$ ion are shown in Fig. 17. All four of them are characterized by potential wells deep enough to keep a significant part of the wave function bound and oscillating with amplitudes and in an energy range comparable to the experimental values. These states dissociate to the same KER of about $11 \mathrm{eV}$,

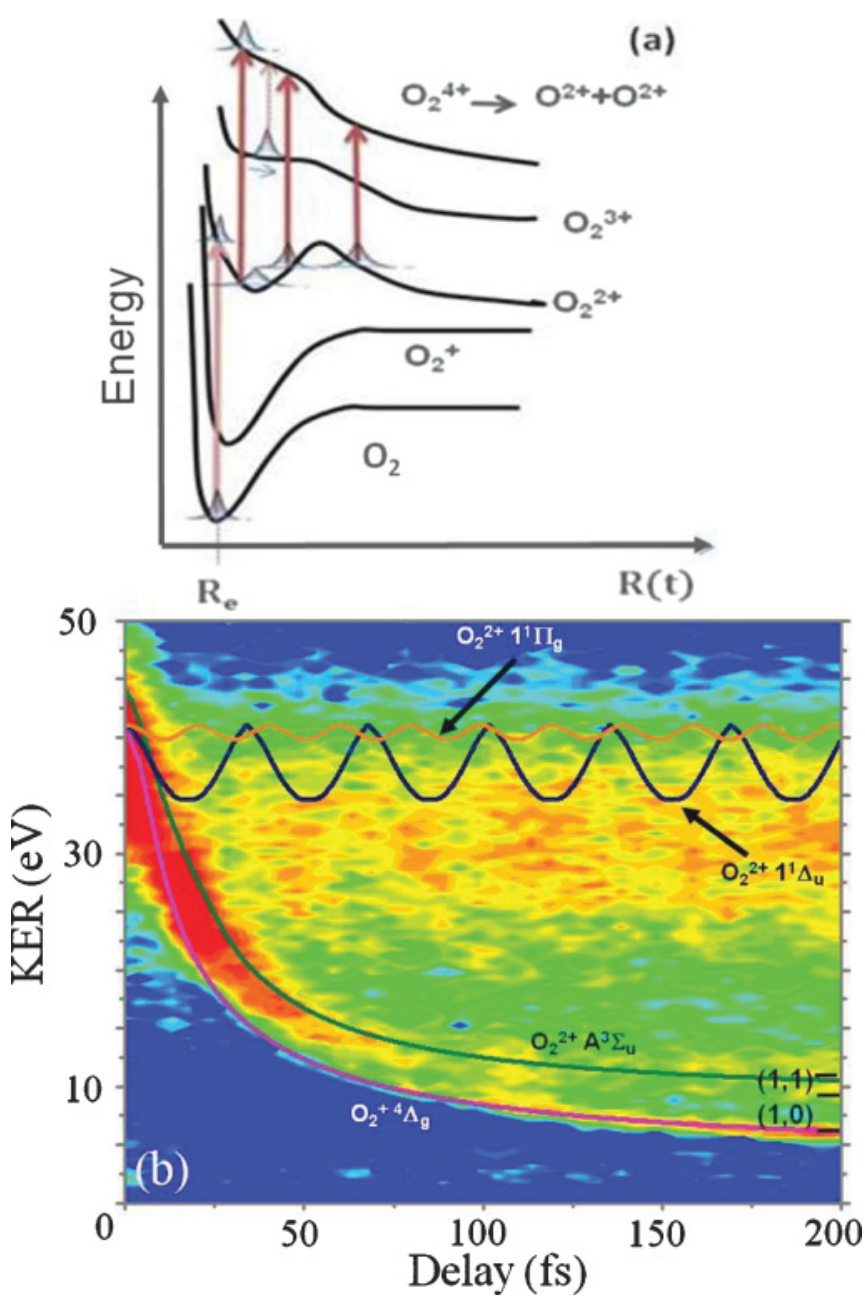

FIG. 15. (Color online) (a) Schematic presentation of wavepacket motion on potential curves of the $\mathrm{O}^{+}$and $\mathrm{O}^{2+}$ ions. (b) Classically calculated time-dependent KER traces superimposed over the experimental spectrum. Each line corresponds to a certain intermediate electronic state of a molecular ion as indicated. The laser parameters are the same as in Fig. 5.

which agrees very well with the corresponding features in the experimental spectra.

\section{Carbon monoxide}

Figure 18 shows the time-dependent KER spectra for four final breakup channels: $\mathrm{C}^{+}+\mathrm{O}^{+}(1,1), \mathrm{C}^{2+}+\mathrm{O}^{+}(2,1)$, $\mathrm{C}^{+}+\mathrm{O}^{2+}(1,2)$, and $\mathrm{C}^{2+}+\mathrm{O}^{2+}(2,2)$. For the $(1,1)$ channel [Fig. 18(a)] there is no visible kinetic energy variation with time. Both $(2,1)$ and $(1,2)$ channels [Figs. 18(b) and 18(c)] reveal features that are familiar from previous experiments with homonuclear diatomic molecules $\mathrm{D}_{2}, \mathrm{~N}_{2}, \mathrm{O}_{2}$ [7], [13]. However, the $\mathrm{C}^{2+}+\mathrm{O}^{+}$channel is more abundant than the $\mathrm{C}^{+}+\mathrm{O}^{2+}$ channel because it is easier to make $\mathrm{C}^{2+}$ than $\mathrm{O}^{2+}$ due to its lower ionization potential. The time-dependent KER for the $\mathrm{C}^{2+}+\mathrm{O}^{2+}$ fragmentation channel is shown in Fig. 18(d).

One can distinguish dissociation traces going down to low energy, but most of the events are concentrated in the region between 22 and $30 \mathrm{eV}$ and along dissociative traces up to 30 fs delay. Similar to $\mathrm{N}_{2}$ and $\mathrm{O}_{2}$, time-dependent KER 

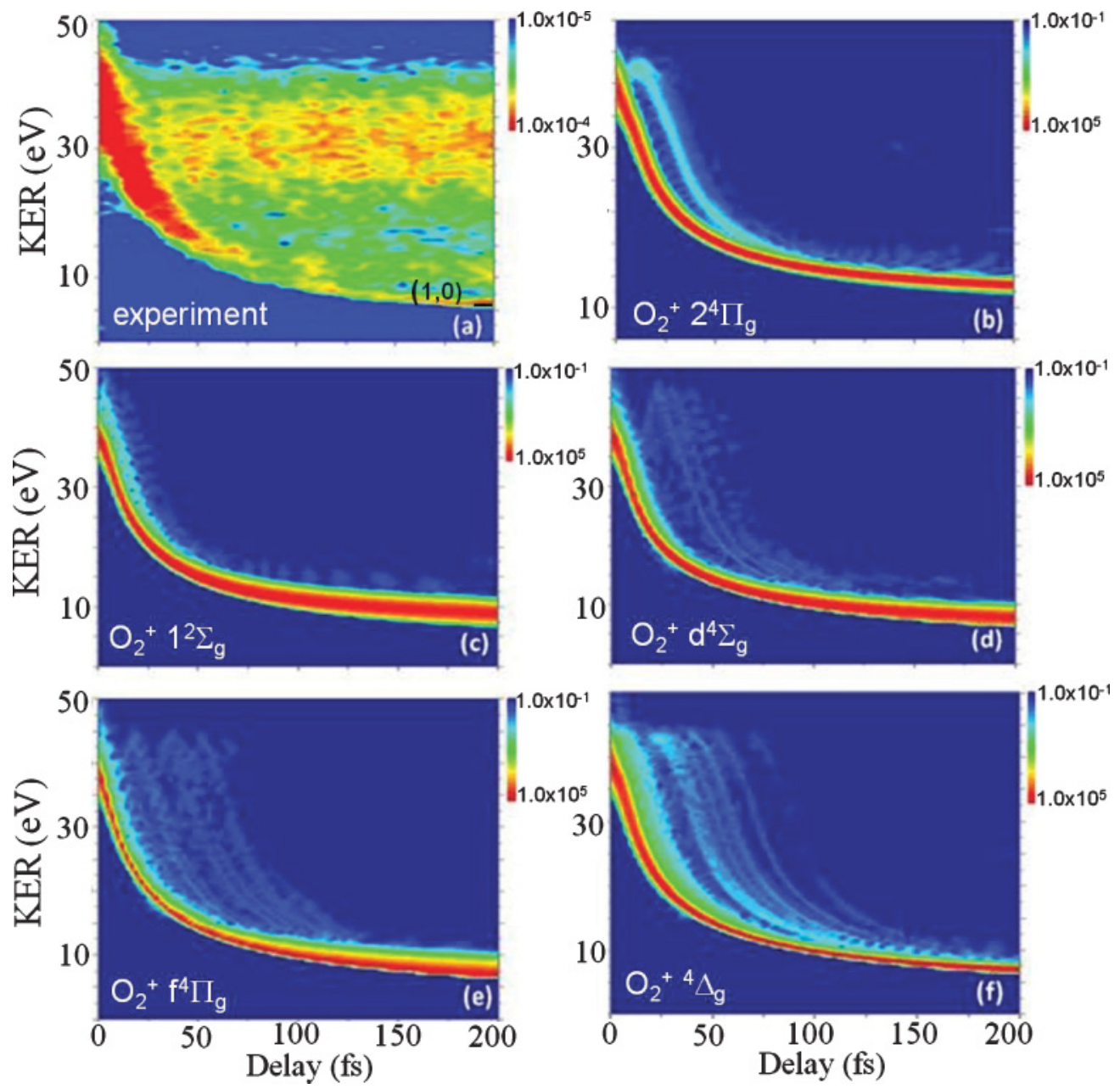

FIG. 16. (Color online) Simulated time-dependent KER spectra for various possible dissociation pathways. (a) The experimental KER spectrum is shown for comparison. (b) $\mathrm{O}_{2} \rightarrow \mathrm{O}_{2}^{+}\left(2^{4} \Pi_{g}\right) \rightarrow \mathrm{O}_{2}^{4+} \rightarrow \mathrm{O}^{2+}+\mathrm{O}^{2+}$. (c) $\mathrm{O}_{2} \rightarrow \mathrm{O}_{2}^{+}\left(1^{2} \Sigma_{g}\right) \rightarrow \mathrm{O}_{2}^{4+} \rightarrow \mathrm{O}^{2+}+\mathrm{O}^{2+}$. (d) $\mathrm{O}_{2} \rightarrow$ $\mathrm{O}_{2}^{+}\left(d^{4} \Sigma_{g}\right) \rightarrow \mathrm{O}_{2}^{4+} \rightarrow \mathrm{O}^{2+}+\mathrm{O}^{2+}$. (e) $\mathrm{O}_{2} \rightarrow \mathrm{O}_{2}^{+}\left(f^{4} \Pi_{g}\right) \rightarrow \mathrm{O}_{2}^{4+} \rightarrow \mathrm{O}^{2+}+\mathrm{O}^{2+}$. (f) $\mathrm{O}_{2} \rightarrow \mathrm{O}_{2}^{+}\left({ }^{4} \Delta_{g}\right) \rightarrow \mathrm{O}_{2}^{4+} \rightarrow \mathrm{O}^{2+}+\mathrm{O}^{2+}$. The laser parameters are the same as in Fig. 5.

spectra for $\mathrm{CO}$ are taken starting from negative delays and show some asymmetry due to the effect of different beam and pulse parameters for pump and probe pulses. To interpret the experimental results, we applied our QM model to simulate time-dependent KER spectra for CO.

For the simulation of time-dependent KER for CO we used the same model that was described for nitrogen and oxygen molecules before. We modeled the final breakup channel for which the best statistics were obtained in our experiment: $\mathrm{CO}^{3+} \rightarrow \mathrm{C}^{2+}+\mathrm{O}^{+}$. We approximated the breakup on the $\mathrm{CO}^{3+}$ curve based on the $2 / R$ Coulomb potential and assumed that it proceeds via $\mathrm{CO}^{+}$and $\mathrm{CO}^{2+}$ intermediate charge states. The relevant potential energy curves for $\mathrm{CO}$, $\mathrm{CO}^{+}$, and $\mathrm{CO}^{2+}$ are presented in Fig. 19 [37-40]. The calculated time-dependent KER spectra for the $(2,1)$ final channel together with the experimental spectra are shown in Figs. 20-22.

Panels (b), (c), and (d) of Fig. 20 show, respectively, the spectra for wave packets evolving on the $E^{2} \Pi, D^{2} \Pi$, and $F^{2} \Pi$ electronic states of the $\mathrm{CO}^{+}$molecular ion. The initial wave packet is obtained in the Franck-Condon approximation as a projection of the $X^{1} \Sigma^{+}$ground state nuclear wave function of CO. It is centered at 2.1 a.u. These three $\mathrm{CO}^{+}$states are all purely dissociative and converge to different asymptotic energies. The trace in Fig. 20(c), corresponding to the $D^{2} \Pi$ state, goes down to approximately $7 \mathrm{eV}$ at $200 \mathrm{fs}$ delay and follows one of the dissociative traces in the experimental spectra, while the other two have higher asymptotic energies.

The states $A^{2} \Pi$ and $C^{2} \Sigma$, corresponding to panels (b) and (c) in Fig. 21, lead to dissociation of the wave packet with fragment energies slightly below $5 \mathrm{eV}$ at $200 \mathrm{fs}$, similar to the spectrum in Fig. 21(a), and keep a part of the wave packet bouncing in a potential well. The oscillating part, though, does not quite fit any feature that we see in the experiment. The spectrum in Fig. 22(c), in contrast, models the wave-packet behavior on the potential curve of the $b^{1} \Pi$ electronic state of $\mathrm{CO}^{2+}$ and reveals oscillating and dissociating features closely resembling those in the experimental spectrum. Though we are not able to resolve clearly the oscillating structure in the energy region between 13 and $18 \mathrm{eV}$ in the experimental plot, we see that in the simulated spectra the bound part falls within the same energy range. Dissociation on the $b^{1} \Pi$ [Fig. 22(c)] and $c^{1} \Delta$ [Fig. 22(d)] potential energy curves of $\mathrm{CO}^{2+}$ produces a time-dependent KER spectrum which 

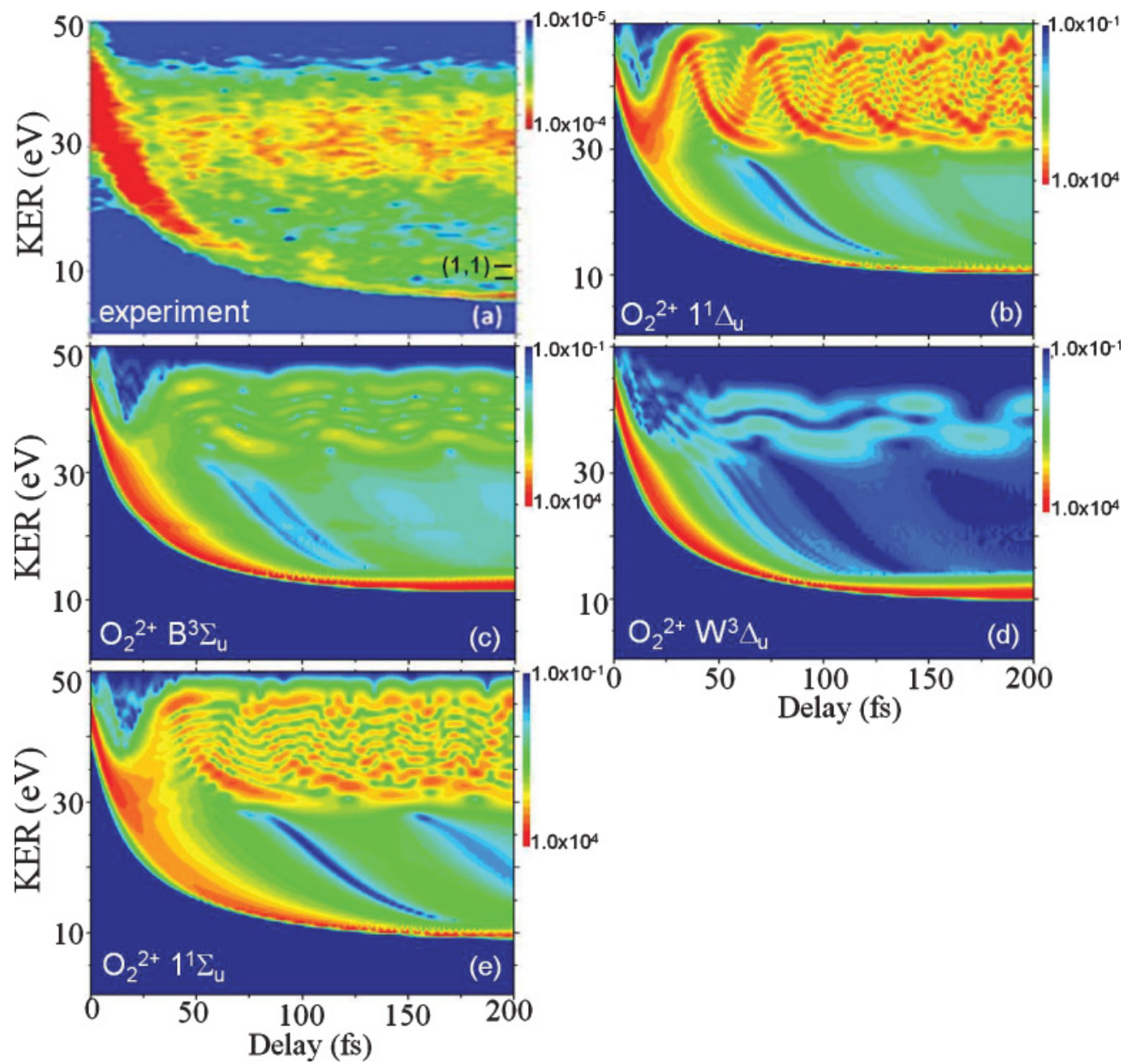

FIG. 17. (Color online) Simulated time-dependant KER spectra for various possible dissociation pathways. (a) The experimental KER spectrum is shown for comparison. (b) $\mathrm{O}_{2} \rightarrow \mathrm{O}_{2}^{2+}\left(1^{1} \Delta_{u}\right) \rightarrow \mathrm{O}_{2}^{4+} \rightarrow \mathrm{O}^{2+}+\mathrm{O}^{2+}$. (c) $\mathrm{O}_{2} \rightarrow \mathrm{O}_{2}^{2+}\left(B^{3} \Sigma_{u}\right) \rightarrow \mathrm{O}_{2}^{4+} \rightarrow \mathrm{O}^{2+}+\mathrm{O}^{2+}$, (d) $\mathrm{O}_{2} \rightarrow$ $\mathrm{O}_{2}^{2+}\left(W^{3} \Sigma_{u}\right) \rightarrow \mathrm{O}_{2}^{4+} \rightarrow \mathrm{O}^{2+}+\mathrm{O}^{2+}$. (e) $\mathrm{O}_{2} \rightarrow \mathrm{O}_{2}^{2+}\left(1^{1} \Sigma_{u}\right) \rightarrow \mathrm{O}_{2}^{4+} \rightarrow \mathrm{O}^{2+}+\mathrm{O}^{2+}$. The laser parameters are the same as in Fig. 5 .

fits the experimental trace with the $7 \mathrm{eV}$ asymptotic energy. The calculated spectrum in Fig. 22(b) for the wave-packet evolution on the $\mathrm{CO}^{2+}\left(A^{2} \Pi\right)$ potential energy curve does not reproduce the experimental features.

\section{SUMMARY}

We investigated the nuclear dynamics in molecular nitrogen, oxygen, and carbon monoxide following their interaction with intense few-cycle laser pulses. By measuring the time-dependent fragment KER spectra after Coulomb explosion in different breakup channels, we observed the process of molecular dissociation as it occurred in real time. We simulated our experimental result using classical and quantum mechanical models. Good agreement between the experiments and simulations allows us to identify transient electronic and charge states and pathways involved in the dynamics. For both $\mathrm{N}_{2}$ and $\mathrm{O}_{2}$ we were able to precisely time the localization dynamics of an unpaired electron in a dissociating molecule, which occurs just 20 fs after the initial ionization. We have also determined that this localization takes place when the internuclear distance increases to about 2.5 times the equilibrium bond length. This distance is the same as the critical distance for enhanced ionization deduced from indirect measurements, thus confirming experimentally that electron localization is an essential step in the enhanced ionization mechanism and that it is a common feature among homonuclear diatomic molecules.

\section{ACKNOWLEDGMENTS}

This work was supported by Chemical Sciences, Geosciences and Biosciences Division, Office of Basic Energy Sciences, US Department of Energy and by the National Science Foundation.

\section{APPENDIX A: CLASSICAL MODEL}

Considering one electronic state $V(R)$ of the molecular ion at a time, we solve Newton's equations of motion for a particle with the reduced mass $\mu=m_{1} m_{2} /\left(m_{1}+m_{2}\right)$ of the two nuclei at time $t=0$ at the equilibrium position $R_{e}$ of the neutral molecule, subject to the initial conditions $R(t=0)=R_{e}$ and $d R / d t(t=0)=0$. We then calculate for all internuclear distances $R$ of the molecular ion the acceleration

$$
a=-\frac{1}{\mu} \frac{d V(R)}{d R},
$$



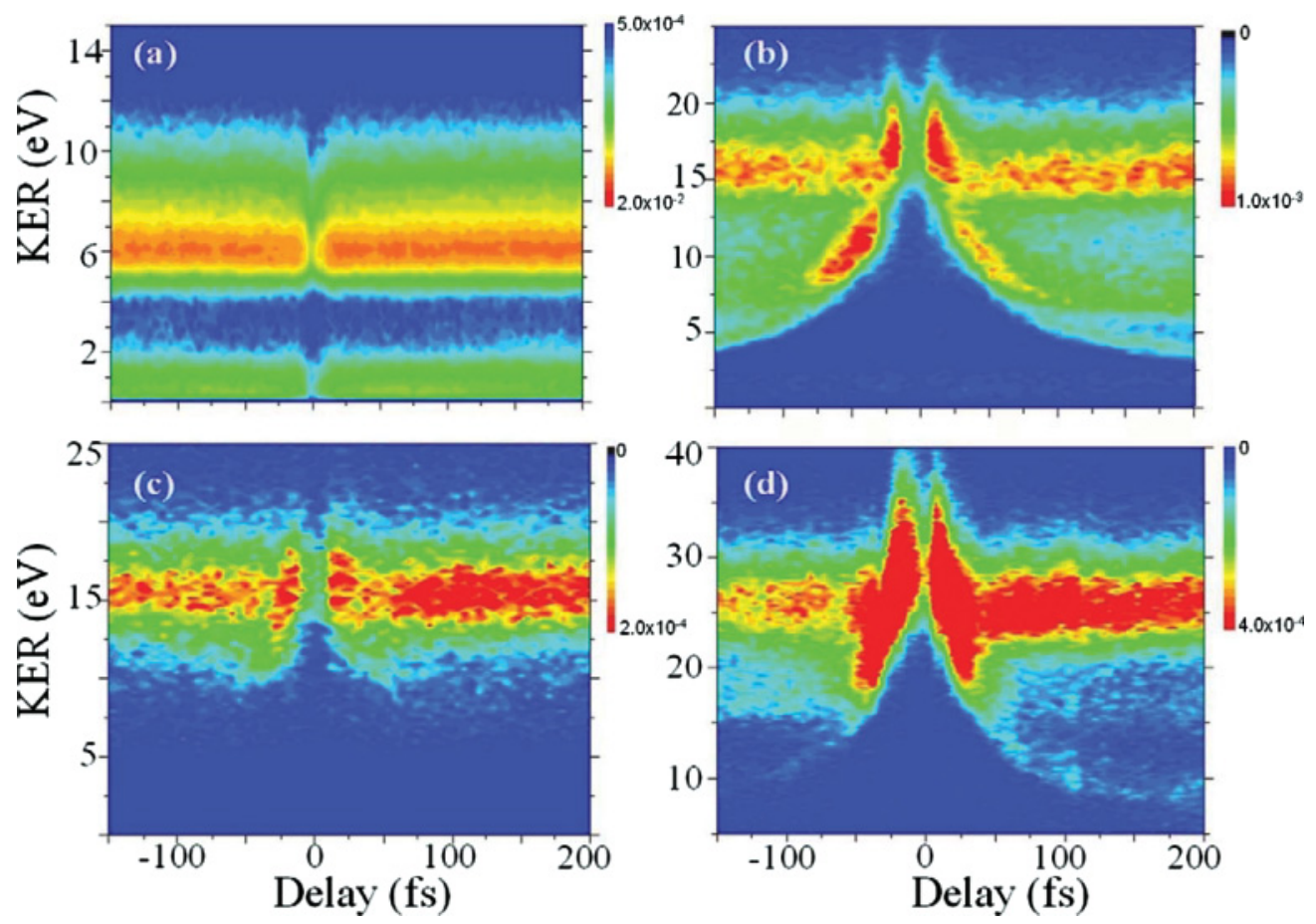

FIG. 18. (Color online) The experimental time-dependent KER spectra for final the breakup channels (a) $\mathrm{CO}^{2+} \rightarrow \mathrm{C}^{+}+\mathrm{O}^{+}$, (b) $\mathrm{CO}^{3+} \rightarrow$ $\mathrm{C}^{2+}+\mathrm{O}^{+}$, (c) $\mathrm{CO}^{3+} \rightarrow \mathrm{C}^{+}+\mathrm{O}^{2+}$, and (d) $\mathrm{CO}^{4+} \rightarrow \mathrm{C}^{2+}+\mathrm{O}^{2+}$. The pump (probe) pulse has a duration of $8 \mathrm{fs}(8 \mathrm{fs})$ and an intensity of $9 \times 10^{14} \mathrm{~W} / \mathrm{cm}^{2}\left(14 \times 10^{14} \mathrm{~W} / \mathrm{cm}^{2}\right)$.

kinetic energy

$$
E_{\mathrm{kin}}(R)=V(R)-V\left(R_{e}\right),
$$

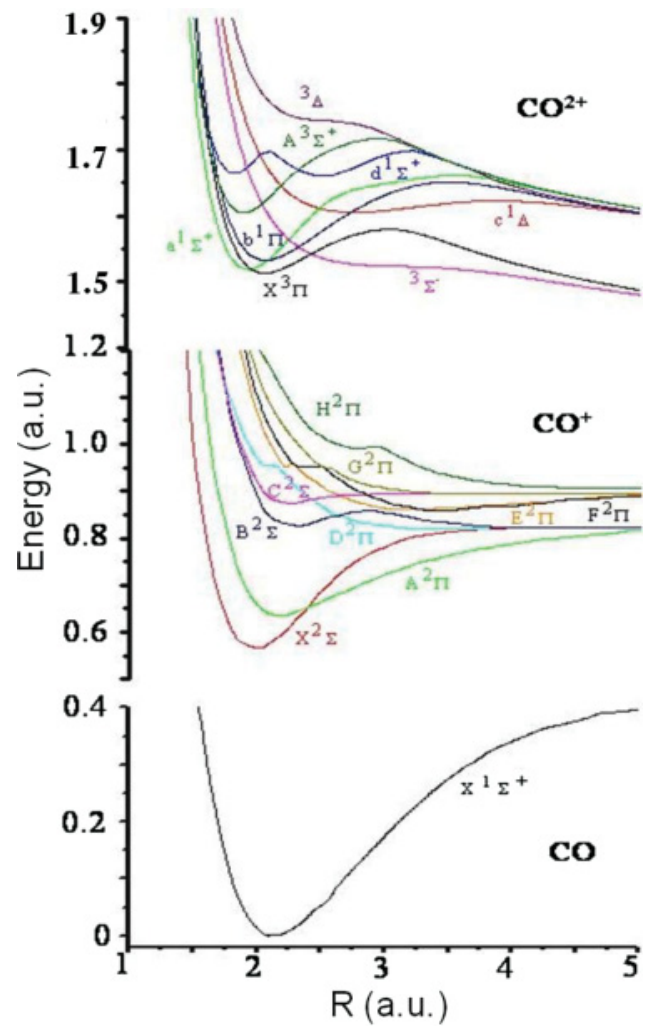

FIG. 19. (Color online) Selected potential energy curves for the $\mathrm{CO}$ molecule and $\mathrm{CO}^{+}$and $\mathrm{CO}^{2+}$ ions [37-40]. velocity

$$
v(R)=\sqrt{2 E_{\mathrm{kin}} / \mu},
$$

and time interval it takes to change the position by $\Delta R$

$$
\Delta t(R)=\frac{-v(R)+\sqrt{v^{2}(R)+2 a(R) \Delta R}}{a(R)}
$$

To calculate the kinetic energy release we add the Coulombexplosion energy for ion fragments at the internuclear distance $R$,

$$
\begin{gathered}
E_{\mathrm{CE}}(R(t))=\frac{q_{1} q_{2}}{R(t)}, \\
E_{\mathrm{KER}}(R(t))=E_{\mathrm{kin}}(R(t))+E_{\mathrm{CE}}(R(t)) .
\end{gathered}
$$

Using an equidistant numerical grid $\left(R_{1}, R_{2}, \ldots, R_{n}\right)$ with sufficiently small spatial steps $\Delta R$ (we use a value of 0.005 a.u. in our numerical calculations), we apply the trapezoid rule in order to approximate

$$
\begin{gathered}
a_{n}=a\left(R_{n}-\Delta R / 2\right)=-\frac{1}{\mu} \frac{V\left(R_{n}\right)-V\left(R_{n-1}\right)}{\Delta R}, \\
\Delta t_{n}=\Delta t\left(R_{n}\right)=\frac{-v\left(R_{n-1}\right)+\sqrt{v^{2}\left(R_{n-1}\right)+2 a_{n} \cdot \Delta R}}{a_{n}}
\end{gathered}
$$

and

$$
t_{n}=\sum_{i=1}^{n} \Delta t_{i}
$$

To compare our classical propagation results with measured KERs, we plot the calculated curves $\operatorname{KER}\left(t_{n}\right)$ on top of the experimental spectra. 

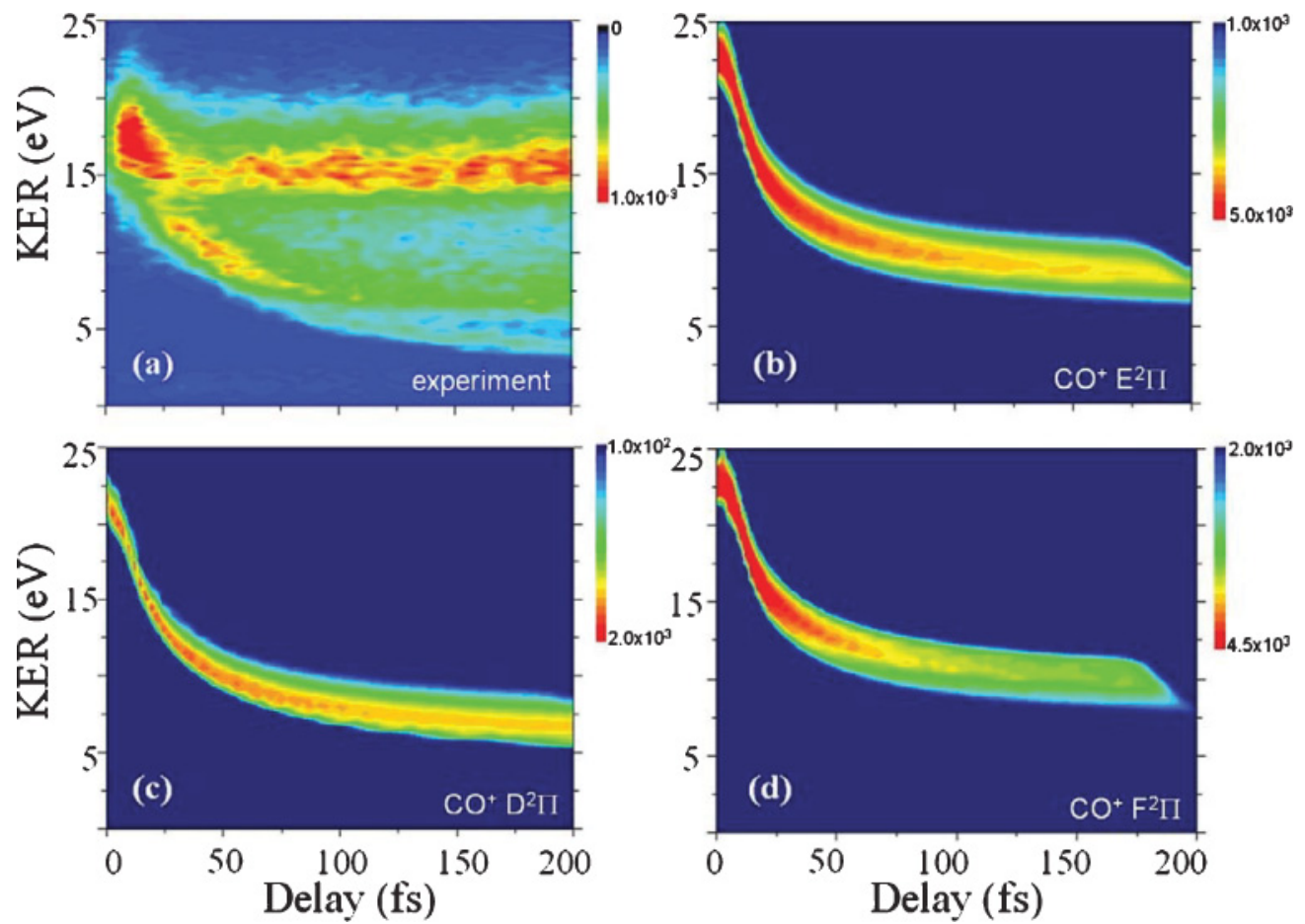

FIG. 20. (Color online) Simulated time-dependent KER spectra for the $\mathrm{CO} \rightarrow \mathrm{C}^{2+}+\mathrm{O}^{+}$final fragmentation channel. (a) The experimental KER spectrum is shown for comparison; simulated results for different pathways: (b) $\mathrm{CO} \rightarrow \mathrm{CO}^{+}\left(E^{2} \Pi\right) \rightarrow \mathrm{CO}^{3+} \rightarrow \mathrm{C}^{2+}+\mathrm{O}^{+}$, (c) $\mathrm{CO}^{2} \rightarrow$ $\mathrm{CO}^{+}\left(D^{2} \Pi\right) \rightarrow \mathrm{CO}^{3+} \rightarrow \mathrm{C}^{2+}+\mathrm{O}^{+}$, and $(\mathrm{d}) \mathrm{CO} \rightarrow \mathrm{CO}^{+}\left(F^{2} \Pi\right) \rightarrow \mathrm{CO}^{3+} \rightarrow \mathrm{C}^{2+}+\mathrm{O}^{+}$. Laser parameters are the same as in Fig. 18 .
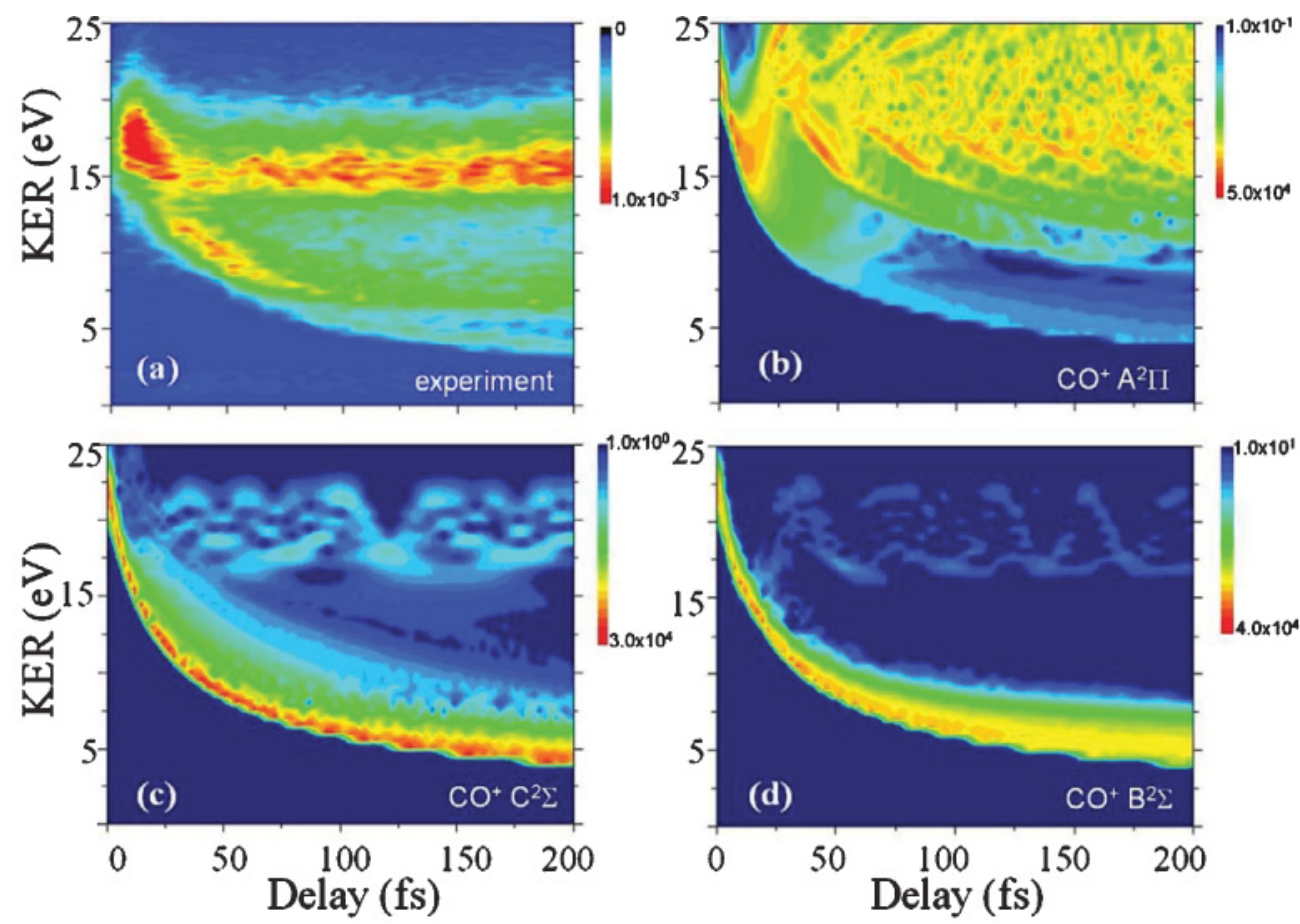

FIG. 21. (Color online) Simulated time-dependent KER spectra for the $\mathrm{CO} \rightarrow \mathrm{C}^{2+}+\mathrm{O}^{+}$final fragmentation channel. (a) The experimental KER spectrum is shown for comparison; simulated results for different pathways: (b) $\mathrm{CO} \rightarrow \mathrm{CO}^{+}\left(A^{2} \Pi\right) \rightarrow \mathrm{CO}^{3+} \rightarrow \mathrm{C}^{2+}+\mathrm{O}^{+}$, (c) $\mathrm{CO} \rightarrow$ $\mathrm{CO}^{+}\left(C^{2} \Sigma\right) \rightarrow \mathrm{CO}^{3+} \rightarrow \mathrm{C}^{2+}+\mathrm{O}^{+}$, (d) $\mathrm{CO} \rightarrow \mathrm{CO}^{+}\left(B^{2} \Sigma\right) \rightarrow \mathrm{CO}^{3+} \rightarrow \mathrm{C}^{2+}+\mathrm{O}^{+}$. Laser parameters are the same as in Fig. 18. 

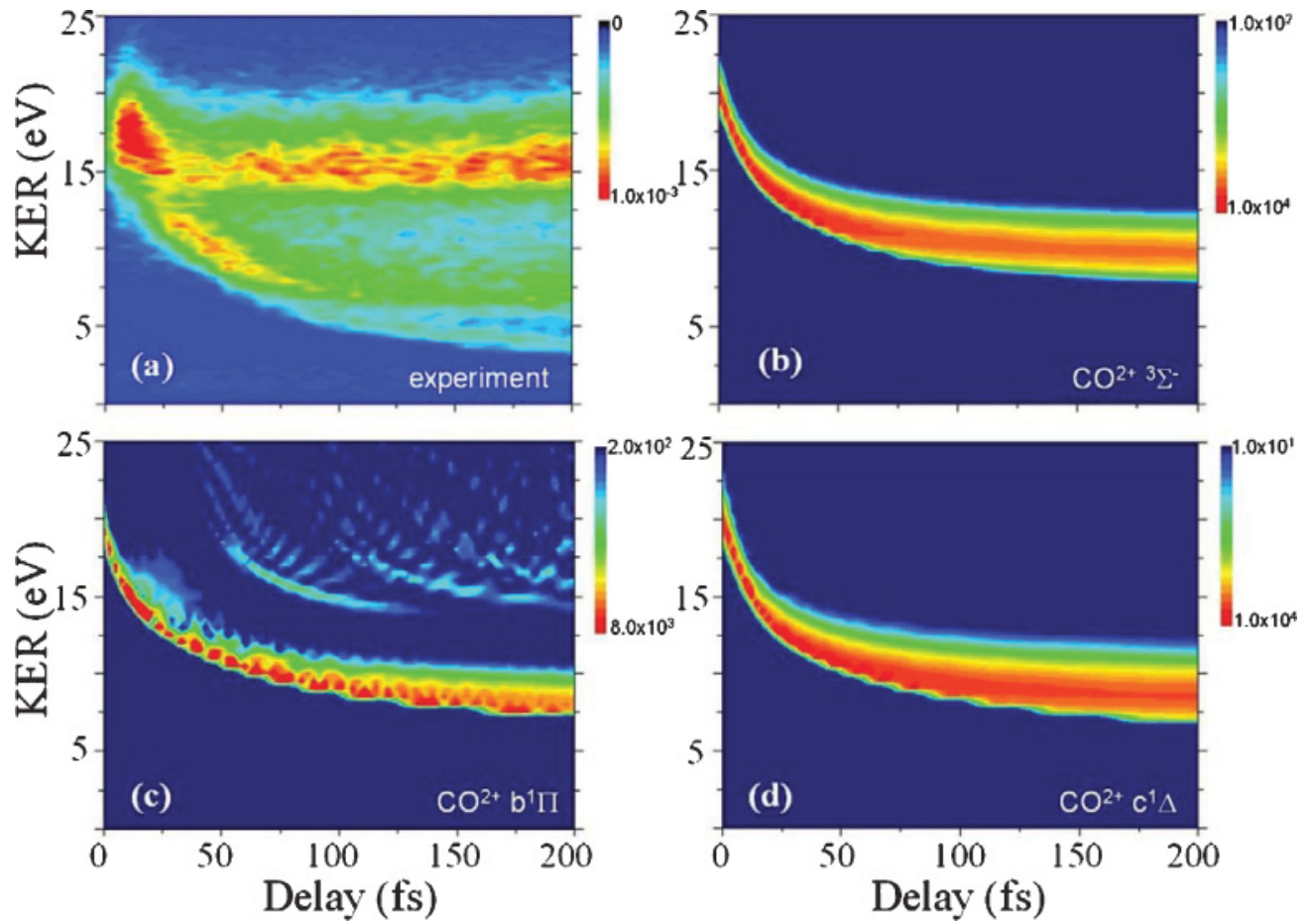

FIG. 22. (Color online) Simulated time-dependent KER spectra for the $\mathrm{CO} \rightarrow \mathrm{C}^{2+}+\mathrm{O}^{+}$final fragmentation channel. (a) The experimental KER spectrum is shown for comparison; simulated results for different pathways: (b) $\mathrm{CO} \rightarrow \mathrm{CO}^{2+}\left({ }^{3} S^{-}\right) \rightarrow \mathrm{CO}^{3+} \rightarrow \mathrm{C}^{2+}+\mathrm{O}^{+}$, (c) $\mathrm{CO} \rightarrow$ $\mathrm{CO}^{2+}\left(b^{1} \Pi\right) \rightarrow \mathrm{CO}^{3+} \rightarrow \mathrm{C}^{2+}+\mathrm{O}^{+}$, (d) $\mathrm{CO} \rightarrow \mathrm{CO}^{2+}\left(c^{1} \Delta\right) \rightarrow \mathrm{CO}^{3+} \rightarrow \mathrm{C}^{2+}+\mathrm{O}^{+}$. Laser parameters are the same as in Fig. 18.

\section{APPENDIX B: QUANTUM MECHANICAL MODEL}

We solve the TDSE,

$$
i \frac{\partial}{\partial t} \Psi(R, t)=\hat{H} \Psi(R, t),
$$

on a numerical grid using the Crank-Nicholson scheme (we use atomic unit unless indicated otherwise). Excluding laserinduced couplings between Born-Oppenheimer (BO) electronic states of the molecular ion, the potential energy operator $\hat{V}$ in the Hamiltonian $\hat{H}=\hat{T}+\hat{V}(R)$ becomes diagonal, such that $\hat{H=\hat{T}}+V(R) \delta_{i j}$, where $V(R)$ is the BO potential for a given electronic state of the molecular ion. $\hat{T}$ is the kinetic energy operator

$$
\hat{T}=-\frac{1}{2 \mu} \frac{\partial^{2}}{\partial R^{2}}
$$

Equation (B.1) can be formally integrated as

$$
\Psi(R, t)=\hat{\tau} \exp \left(-i \int_{t_{0}}^{t} \hat{H} d t^{\prime}\right) \Psi\left(R, t_{0}\right),
$$

where $\hat{\tau}$ is the time-ordering operator and $\Psi\left(R, t_{0}\right)$ the initial wave packet which we choose to be the wave function of the neutral molecule in its electronic and vibrational ground state.

For sufficiently small time steps $\Delta t$ (we use $\Delta t=0.1$ a.u. in our numerical calculations), we apply the Cayley scheme [34] and evaluate

$$
\begin{gathered}
\Psi\left(t_{n+1}\right)=\exp (-i \hat{H} \Delta t) \Psi\left(t_{n}\right) \\
\Psi\left(t_{n+1}\right) \approx\left(1-i \hat{H} \frac{\Delta t}{2}\right) /\left(1+i \hat{H} \frac{\Delta t}{2}\right) \Psi\left(t_{n}\right) .
\end{gathered}
$$

Using a three-point finite differencing, we represent the kinetic energy operator in terms of the tridiagonal matrix

$$
\left(T_{R}\right)_{i j}=-\frac{1}{2 \mu \Delta R^{2}}\left(\delta_{i, j-1}-2 \delta_{i, j}+\delta_{i, j+1}\right)
$$

In our QM model the short laser pulse does not couple the potential curves, but only removes electrons, thus transferring the nuclear wave packet from one potential curve to another without affecting its shape (Frank-Condon transition). The following time propagation is carried out without a laser field. After some delay, the probe pulse removes additional electrons, transferring the wave packet to the final potential curve, which is assumed to be Coulombic. Since electrons are not removed instantaneously by the laser pulse of a finite width, we also propagate the wave packet on an appropriate trial-intermediate state. The time of this propagation is a parameter in our model which we vary to match the calculated KER spectrum with the experimental one.

To calculate KER as a function of time, we project $\Psi(R, t)$ onto the Coulomb radial $(l=0)$ wave function $\Phi_{c}(E, R)$. The KER then is given by Franck-Condon factor [41] as

$$
E_{\mathrm{KER}}(t)=\left|\int_{0}^{\infty} \Phi_{C}^{*}(E, R) \Psi(R, t) d R\right|^{2} .
$$

We performed the described calculations by adopting the FORTRAN 90 code developed in [22-24]. 
[1] P. H. Bucksbaum, A. Zavriyev, H. G. Muller, and D. W. Schumacher, Phys. Rev. Lett. 64, 1883 (1990).

[2] M. Winter, R. Schmidt, and U. Thumm, New J. Phys. 12, 023020 (2010); Phys. Rev. A 80, 031401(R) (2009).

[3] M. Magrakvelidze, F. He, T. Niederhausen, I. V. Litvinyuk, and U. Thumm, Phys. Rev. A 79, 033410 (2009).

[4] U. Thumm, T. Niederhausen, and B. Feuerstein, Phys. Rev. A 77, 063401 (2008); B. Feuerstein, T. Ergler, A. Rudenko, K. Zrost, C.D. Schröter, R. Moshammer, J. Ullrich, T. Niederhausen, and U. Thumm, Phys. Rev. Lett. 99, 153002 (2007).

[5] T. Zuo and A. D. Bandrauk, Phys. Rev. A 52, R2511 (1995).

[6] H. Niikura, F. Légaré, R. Hasbani, A.D. Bandrauk, M.Y. Ivanov, D. M. Villeneuve, and P. B. Corkum, Nature (London) 417, 917 (2002).

[7] A. S. Alnaser et al., Phys. Rev. Lett. 93, 183202 (2004).

[8] K. Miyazaki, T. Shimizu, and D. Normand, J. Phys. B 37, 753 (2004).

[9] J. Muth-Böhm, A. Becker, and F. H. M. Faisal, Phys. Rev. Lett. 85, 2280 (2000).

[10] T. Seideman, M.Y. Ivanov, and P. B. Corkum, Phys. Rev. Lett. 75, 2819 (1995).

[11] C. Guo, M. Li, and G. N. Gibson, Phys. Rev. Lett. 82, 2492 (1999).

[12] J. P. Nibarger, S. V. Menon, and G. N. Gibson Phys. Rev. A 63, 053406 (2001).

[13] S. Voss, A. S. Alnaser, X.-M. Tong, C. Maharjan, P. Ranitovic, B. Ulrich, B. Shan, Z. Chang, C. D. Lin, and C. L. Cocke, J. Phys. B 37, 4239 (2004).

[14] H. Ren, R. Ma, J. Chen, X. Li, H. Yang, and Q. Gong, J. Phys. B 36, 2179 (2003).

[15] F. Krausz and M. Y. Ivanov, Rev. Mod. Phys. 81, 163 (2009).

[16] R. Wester, D. Schwalm, A. Wolf, and D. Zaifman, in ManyParticle Quantum Dynamics in Atomic and Molecular Fragmentation, edited by J Ullrich and V. P. Shevelko (Springer, Heidelberg, 2003), p. 411.

[17] J. Ullrich, R. Moshammer, R. Dörner, O. Jagutzki, V. Mergel, H. Schmidt-Böcking, and L. Spielberger, J. Phys. B 30, 2917 (1997)

[18] A. S. Alnaser, X. M. Tong, T. Osipov, S. Voss, C. M. Maharjan, B. Shan, Z. Chang, and C. L. Cocke, Phys. Rev. A 70, 023413 (2004).

[19] E. Thulstrup and A. Andersen, J. Phys. B 8, 965 (1975).

[20] T. Aoto, K. Ito, Y. Hikosaka, A. Shibasaki, R. Hirayama, N. Yamamono, and E. Miyoshi, J. Chem. Phys. 124, 234306 (2006).
[21] A.D. Bandrauk, D. G. Musaev, and K. Morokuma, Phys. Rev. A 59, 4309 (1999).

[22] B. Feuerstein and U. Thumm, Phys. Rev. A 67, 043405 (2003); J. Phys. B 36, 707 (2003).

[23] H. S. Chakraborty, T. Niederhausen, and U. Thumm, Phys. Rev. A 70, 052903 (2004)

[24] T. Niederhausen and U. Thumm, Phys. Rev. A 77, 013407 (2008); T. Niederhausen, Ph.D. thesis, Kansas State University, 2007, [http://jrm.phys.ksu.edu/theses].

[25] I. A. Bocharova, Ph.D. thesis, Kansas State University, 2009, [http://jrm.phys.ksu.edu/theses.html\#Bocharova-PhD].

[26] P. B. Corkum, Phys. Rev. Lett. 71, 1994 (1993)

[27] A. S. Alnaser, T. Osipov, E. P. Benis, A. Wech, B. Shan, C. L. Cocke, X. M. Tong, and C. D. Lin, Phys. Rev. Lett. 91, 163002 (2003).

[28] C. Guo, M. Li, J. P. Nibarger, and G. N. Gibson, Phys. Rev. A 61, 033413 (2000).

[29] A. S. Alnaser, M. Zamkov, X. M. Tong, C. M. Maharjan, P. Ranitovic, C. L. Cocke, and I. V. Litvinyuk, Phys. Rev. A 72, 041402 (2005).

[30] I. V. Litvinyuk, F. Légaré, P. W. Dooley, D. M. Villeneuve, P. B. Corkum, J. Zanghellini, A. Pegarkov, C. Fabian, and T. Brabec, Phys. Rev. Lett. 94, 033003 (2005).

[31] S. De, I. A. Bocharova, M. Magrakvelidze, D. Ray, W. Cao, B. Bergues, U. Thumm, M. F. Kling, I. V. Litvinyuk, and C. L. Cocke, Phys. Rev. A 82, 013408 (2010).

[32] N. H. F. Beebe, E. W. Thultsrup, and A. Andersen, J. Chem Phys. 642080 (1976).

[33] M. Lindqvist, D. Edvardsson, P. Baltzer, M. Larson, and B. Wannberg, J. Phys. B 29, 499 (1996).

[34] D. Edvardsson, S. Lunell, F. Rakowitz, C. M. Marian, and L. Karlsson, Chem. Phys. 229, 203 (1998).

[35] A. Mitrushenkov, P. Palmieri, G. Chambaud, and P. Rosmus, Chem. Phys. Lett. 378, 463 (2003).

[36] A. A. Wills, A. A. Cafolla, and J. Comer, J. Phys. B 24, 3989 (1991).

[37] W. Coughran, J. Rose, T.-I. Shibuya, and V. McKoy, J. Chem. Phys. 58, 2699 (1973).

[38] W. Li, S. A. Lahankar, C. Huang, P. S. Shternin, O. S. Vasyutinskii, and A. G. Suits, Phys. Chem. Chem. Phys. 8, 2950 (2006).

[39] P. Lablanque et al., Phys. Rev. A 40, 5673 (1989).

[40] T. Šedicová, P. R. Žďánská, and V. Špirko, J. Chem. Phys. 124, 214303 (2006).

[41] S. Chelkowski, P. B. Corkum, and A. D. Bandrauk, Phys. Rev. Lett. 82, 3416 (1999). 